Submitted to Physical Review B

\title{
First Principles LCGO calculation of the magneto-optical properties of nickel and iron
}

\author{
Neeraj Mainkar, D. A. Browne and J. Callaway \\ Department of Physics and Astronomy \\ Louisiana State University \\ Baton Rouge, Louisiana 70803
}

(May 10, 2019)

\begin{abstract}
We report a first principles, self-consistent, all electron, linear combination of Gaussian orbitals (LCGO) calculation of a comprehensive collection of magneto-optical properties of nickel and iron based on density functional theory. Among the many magneto-optical effects, we have studied the equatorial Kerr effect for absorption in the optical as well as soft X-ray region, where it is called X-ray magnetic linear dichroism (X-MLD). In the optical region the effect is of the order of $2 \%$ while in the X-ray region it is of the order of $1 \%$ for the incident angles considered. In addition, the polar Kerr effect, X-ray magnetic circular dichroism (X-MCD) and total X-ray absorption at the $\mathrm{L}_{2,3}$ edges, soft X-ray Faraday effect at the $\mathrm{L}_{2,3}$ edges have also been calculated. Our results are in good agreement with experiments and other first principles methods that have been used to calculate some of these properties.
\end{abstract}

PACS numbers: 71.25.Pi, 78.20.Ls

Typeset using REVTEX 


\section{INTRODUCTION}

Although magneto-optical properties of magnetic metals have been known for over a hundred years, 1 it is only in the past couple of decades that vigorous interest has been refocussed on this subject, partly due to their potential for application in the technology of high density data storage 2 Because of advances in laser and tunable synchrotron sources, a variety of different magneto-optical effects including the magneto-optical Kerr effect (MOKE), 目 the Faraday effect, $\mathrm{Q}$ X-ray magnetic circular dichroism for absorption $\mathrm{B}$.

photoemission, 10,11 and X-magnetic linear dichroism in angle resolved photoemission 11,12 have now been extensively studied. The connection between all of these phenomena and the electronic structure of the materials in which they are seen, has long been known.13,14 However, only in recent years has there been an effort to perform first principles band structure calculations of some of these effects. One electron band calculations for bulk and surfaces, alloys and multilayers have been already reported for the polar MOKE, 15 - 19 X-MCD and X-MLD,2025 and the X-ray Faraday effect.27

This paper reports a first principles, self-consistent, all electron calculation of several magneto-optical properties of bulk nickel and iron, using the linear combination of Gaussian orbitals (LCGO) method. These include the equatorial Kerr effect, X-ray magnetic linear dichroism (X-MLD) in absorption at the $2 p$ edges, the soft X-ray Faraday effect at the $2 p$ edges, the X-MCD and absorption spectra at the $2 p$ edges as well as the polar MOKE. The equatorial Kerr effect defined here is the difference in absorption of $p$-polarized light incident obliquely on the magnetized sample in the equatorial geometry, when the sample magnetization is reversed. When observed at core-level edges this effect can be called photoabsorption X-MLD of a new kind to distinguish it from the first kind observed by holding the photon polarization parallel to the $x$-axis and rotating the magnetization from the $x$ to the $z$ axis, 26 the second kind obseved due to the rotation of the photon polarization vector with the magnetization held fixed22 and the one observed in angle resolved photemission.

Our motivation in performing these calculations has been two-fold. Firstly, since it is 
known that these effects depend sensitively on the accuracy of the band structure calculation, we wanted to demonstrate that our modified tight binding method that includes spin-orbit coupling in a very straightforward manner, can produce results at least as accurate as other $a b$-initio electronic structure approaches that apply either the fully relativistic machinery or use more complicated computational procedures to avoid using the Kramers Kronig (KK) transformation. Our MOKE results on nickel and iron essentially prove this point. Secondly, although experiments on the equatorial Kerr effect have been done previously in the optical region 28 no experiments on the above-mentioned photoabsorption X-MLD at the $2 p$ edge of nickel and iron have been reported. It would thus be interesting to see how well our theoretical results for the $2 p$ edge agree with future experiments. A recent experiment on

the soft X-ray Faraday effect at the $2 p$ edges of iron h has also spurred us into calculating this effect using the LCGO method.

We thus begin Section II by outlining in brief the inclusion of spin-orbit coupling to the non-relativistic LCGO method, which is slightly different from the previous LCGO work.29 We also give a brief description of the fast and efficient KK transformation method we have employed in our analysis. In section III we discuss in detail the results of our first principles calculation of the elements of the conductivity tensor and their subsequent use in determining the magneto-optical properties mentioned above. We shall also point out how our method compares with previous theoretical results and with available data.

\section{METHOD OF CALCULATION}

The band structure calculation with spin-orbit coupling included within the LCGO method was first done by Wang and Callawaye 29 for nickel and by Singh, Wang and Callaway for iron 30 However, the exchange potential in those calculations was of the $\mathrm{X} \alpha$ kind and the set of 38 basis functions was also small. Subsequently, the computational procedure was revised considerably, 31 with the exchange potential being replaced by the more accurate vonBarth and Hedin type as parametrized by Rajagopal, Singhal and Kimball, 22 and the basis 
set expanded to a total of 75 functions for $3 d$ transition metals. The method of evaluating the Brillouin zone (BZ) integrals was also improved by using the linear analytic tetrahedron method, 33, 34 and improvements were made in calculating the $K=0$ Fourier component of the Coulomb potential.35 This non-relativistic LCGO method was applied to a host of different elemental magnetic as well as non-magnetic solids over the past several years and a variety of electronic properties such as the Fermi surface, the optical conductivity and the Compton profile were calculated 36 which were in fairly good agreement with experiments. For the $3 \mathrm{~d}$ transition metals the basis set thus consisted of 13 s-type, 10 p-type, 5 d-type and 1 f-type Gaussian orbitals, using published orbitals based on atomic calculations. 15 However, relativistic corrections were completely ignored in these improvements.

With a view to tackling the anisotropic properties of magnetic metals, we have added the spin-orbit coupling, Darwin and relativistic mass-velocity terms to the existing code. As is well-known, the principal advantage to using a Gaussian basis in a first principles tight-binding method is the ability to use analytic expressions for the overlap and several Hamiltonian matrix elements. This is true in the case of the spin orbit interaction term as well. We have used essentially the same approximations used earlier 29 for the spin-orbit coupling term i.e., the principal contribution to the spin-orbit matrix elements is only for those in the $p-p$ and $d-d$ blocks. We also used the same central-cell approximation wherein we retain only spin-orbit matrix elements between those orbitals centered on the same atomic site. The difference between our new approach and theirs is that in Ref. 29 the spin orbit matrix elements were evaluated in reciprocal space and a Fourier sum had to be performed over $K$ vectors to obtain the real space potential. Since the spin-orbit coupling is strongest at smaller distances, this necessitated the use of an asymptotic expansion for the handling of larger $K$ values to obtain a reasonable value for the $K$ summation. We have avoided this completely by evaluating the spin-orbit matrix elements in real space directly. In terms of the Gaussian basis, the expresssion for the spin orbit matrix elements of the $p-p$ block is, 


$$
I_{p}=\frac{\hbar^{2} e^{2} N_{p}}{8 m^{2} c^{2} \alpha}\left[Z-\frac{1}{2 N \Omega} \sum_{i, j}^{n_{b}} \frac{P_{i j} N_{i j} \Gamma\left(\left(l_{i j}+3\right) / 2\right)}{\left(\alpha+\alpha_{i j}\right)^{\frac{l_{i j}+3}{2}}}\right]
$$

while for those of the $d-d$ block is

$$
I_{d}=\frac{\hbar^{2} e^{2} N_{d}}{8 m^{2} c^{2} \alpha^{2}}\left[Z-\frac{1}{2 N \Omega} \sum_{i, j}^{n_{b}} P_{i j} N_{i j}\left\{\frac{\Gamma\left(\left(l_{i j}+3\right) / 2\right)}{\left(\alpha+\alpha_{i j}\right)^{\frac{l_{i j}+3}{2}}}+\frac{\alpha \Gamma\left(\left(l_{i j}+5\right) / 2\right)}{\left(\alpha+\alpha_{i j}\right)^{\frac{l_{i j}+5}{2}}}\right\}\right]
$$

where

$$
P_{i j}=\frac{1}{48 W} \sum_{k} \rho_{i j}^{o c c}(k) g(k)
$$

In the above expressions $N_{p}$ and $N_{d}$ are the product of the normalization constants of the appropriate Gaussian orbitals for the $p-p$ and $d-d$ block respectively, $n_{b}$ is the number of basis functions, $\alpha$ is the sum of the exponents of the Gaussian orbitals, $N$ is the number of unit cells and $\Omega$ is the unit cell volume, $g(k)$ is the weight associated with each $k$ point in the BZ, $l_{i j}$ is the sum of the orbital quantum numbers of the basis functions $i$ and $j$, $W=\sum_{k} g(k)$ is the total weight, and $\Gamma(z)$ is the Gamma function. For the charge density appearing in the Eq. (3) we used the resulting wavefunctions of the non-relativistic, selfconsistent calculation. With spin-orbit coupling included, the new generalized eigenvalue equation of order $150 \times 150$ was diagonalized in $1 / 16$ th of the BZ at only $219 k$ points for fcc nickel and only $125 k$ points for bcc iron, fewer than the number of points used in the previous calculations.

Using the results of the band structure calculation, the elements of the conductivity tensor may be found from the formulas 29 ,

$$
\begin{aligned}
& \sigma_{x x}(\omega)=\frac{i e^{2}}{m^{2} \hbar} \sum_{k} \sum_{l n} \frac{1}{\omega_{n l}(k)}\left[\frac{\left|\Pi_{l n}^{x}\right|^{2}}{\omega-\omega_{n l}(k)+i \delta}+\frac{\left|\Pi_{l n}^{x}\right|^{2}}{\omega+\omega_{n l}(k)+i \delta}\right] \\
& \sigma_{x y}(\omega)=\frac{i e^{2}}{m^{2} \hbar} \sum_{k} \sum_{l n} \frac{1}{\omega_{n l}(k)}\left[\frac{\Pi_{l n}^{x} \Pi_{n l}^{y}}{\omega-\omega_{n l}(k)+i \delta}+\frac{\left(\Pi_{l n}^{x} \Pi_{n l}^{y}\right)^{\star}}{\omega+\omega_{n l}(k)+i \delta}\right]
\end{aligned}
$$

where $l$ goes over the occupied states and $n$ goes over the unoccupied states and $\Pi$ 's are the $k$ dependent matrix elements of the momentum operator.

The usual procedure is to evaluate the real part of $\sigma_{x x}$ and the imagninary part of $\sigma_{x y}$ by replacing the Lorentzian by a delta function in the limit of $\delta$ going to zero. It is then 
customary to keep $\delta$ finite to simulate a finite relaxation time. For calculations of magnetooptical properties, it is then necessary to perform the KK transformations to obtain the imaginary part of $\sigma_{x x}$ and the real part of $\sigma_{x y} .13$ The KK integrals are known to suffer from problems of slow convergence and the necessity of choosing high cutoff values for energy. One approach 15 evaluates the original Kubo formula directly with lifetime effects as a parameter. This method, although accurate, is extremely computationally intensive. We have instead performed the KK transformations through the use of two succesive Fast Fourier transforms. This method is commonly used in studies of infrared intensities of liquids 4 The basis of this method comes from the well known relation that if $F(t)$ is the Fourier transform of $f(\omega)$,

$$
F(t)=\frac{1}{\sqrt{2 \pi}} \int_{-\infty}^{\infty} d \omega f(\omega) e^{-i \omega t}
$$

and $h(\omega)$ is the Hilbert transform of $f(\omega)$,

$$
h(\omega)=\frac{1}{\pi} P \int_{-\infty}^{\infty} d \omega^{\prime} \frac{f\left(\omega^{\prime}\right)}{\omega-\omega^{\prime}}
$$

then

$$
H(t)=-i \operatorname{sgn}(\mathrm{t}) F(t)
$$

where $H(t)$ is the Fourier transform of $h(\omega)$,

$$
H(t)=\frac{1}{\sqrt{2 \pi}} \int_{-\infty}^{\infty} d \omega h(\omega) e^{-i \omega t}
$$

Preliminary tests on known Hilbert transforms using this method yield results that are accurate to better than $1 \%$. The advantages of this method are immense since with sufficient number of points, the method is very accurate and fast. Furthermore, it has the flexibility of incorporating the lifetime effects very conveniently by simply multiplying the right hand side of Eq. (8) by an exponential damping factor,

$$
H(t)=-i \operatorname{sgn}(\mathrm{t}) F(t) e^{-\delta|t|}
$$

The disadvantage of using this method is the same as that which arises when trying to evaluate the KK integral directly, namely, the need to have function values for frequencies 
more than twice the range of frequencies of interest. This is particularly troublesome for functions that don't quickly die down within the energy range of interest. In our problem this requires the values of the momentum matrix elements for states up to $30 \mathrm{eV}$ above the Fermi level. In the following section we shall show our results of using this method for the elements of the conductivity tensor in the optical as well as X-ray region and compare it with non-KK transformed results.

We have applied the results of the foregoing analysis to determine both the polar and the equatorial Kerr effects. For the polar Kerr effect the complex Kerr rotation is given by the relation, 47

$$
\phi=\frac{-\sigma_{x y}}{\sigma_{x x} \sqrt{1+4 \pi i \sigma_{x x} / \omega}}
$$

We have calculated the equatorial Kerr effect in the optical as well as the soft X-ray region. It is however well known that in the equatorial geometry, the reflection coefficient of $p$-polarized light at oblique incidence depends on the direction of magnetization because of its dependence on the off-diagonal component of the conductivity tensor. Thus it is evident that a reversal in magnetization should cause a change in the absorbed intensity of $p$-polarized light. Moreover, since such an effect does not occur for s-polarized light the equatorial Kerr effect can be observed $\$$ even using unpolarized light. Past calculations 48 for the equatorial Kerr effect in the optical region have used expressions that are correct up to first order in $\kappa_{2}$, the off-diagonal component of the dielectric tensor. To calculate the effect in the X-ray region we need the exact expression for the reflection coefficient of $p$-polarized light incident at an angle of incidence $\theta$,

$$
r=\frac{\cos \theta\left[n \kappa_{1} \beta+\kappa_{2} \sin \theta\right]+\sin ^{2} \theta-\kappa_{1}}{\sin ^{2} \theta-\kappa_{1}-\cos \theta\left[n \kappa_{1} \beta+\kappa_{2} \sin \theta\right]}
$$

where $\beta^{2}=1-\sin ^{2} \theta / n^{2}$ and the complex refractive index $n$ is $n^{2}=\kappa_{1}+\kappa_{2}^{2} / \kappa_{1}$ where $\kappa_{1}$ is the diagonal element of the dielectric tensor. From Eq. (12) the absorption $\left(1-|r|^{2}\right)$ can be obtained. 
Lastly, we also calculated the X-ray Faraday rotation for nickel and iron at the $2 p$ edge. Here we have used the standard expression for the Faraday rotation $\theta_{F}$ for a thickness $d$,

$$
\theta_{F}=\frac{\omega d}{2 c} \operatorname{Re}\left[n^{r}-n^{l}\right]
$$

where $n^{r, l}=\sqrt{1+4 \pi i \sigma^{r, l}(\omega) / \omega}$ and $\sigma^{r, l}(\omega)=\sigma_{x x}(\omega) \pm i \sigma_{x y}(\omega)$.

\section{RESULTS AND DISCUSSION}

\section{A. Elements of the conductivity tensor}

Using the results of the band structure calculation, we calculated $\sigma_{x x}^{1}(\omega)$ and $\sigma_{x y}^{2}(\omega)$ in the optical as well as the X-ray region for nickel and iron. For the optical region, we show our results in comparison with experiments and the theoretical results of Ref. 15. For comparison purposes we have not included the Drude term to the diagonal terms in the figures for $\sigma_{x x}(\omega)$. Later, they have been included in the calculation of the magneto-optical effects. Fig. 1(a) shows the results of $\sigma_{x x}^{1}(\omega)$ for nickel. Our theoretical curve seems to be in good agreement with the experiments of Ref. 50. The difference between our results and those of Ref. 15 is due to a different choice for the lifetime. After repeating the calculation with different values of the lifetime parameter, we chose a value of $0.0368 \mathrm{Ry}$ for nickel to give best results for MOKE. We also observe the $1 \mathrm{eV}$ shift at $5.4 \mathrm{eV}$ which has been ascribed to the failure of the LDA in producing some nickel $3 d$ bands.15 In the case of $\omega \sigma_{x y}^{2}(\omega)$ (Fig. 1(b)) our results are similar to those of Ref. 15 but the peaks are more pronounced in our case. Another feature worth noting is the dip near $5.5 \mathrm{eV}$ that is closer to the observed dip in our calculation than theirs. This has a very noticeable effect on the Kerr angle spectra as we shall see later. In the case of iron, our theoretical results for $\sigma_{x x}^{1}(\omega)$ (Fig. 1(c)) compare far better with experiment than those of nickel and agrees well with the results of Ref. 15 . Our theoretical results seem to be closer to the experiments of Ref. 52 and agreement in general can be taken to be quite good. In the case of $\omega \sigma_{x y}^{2}(\omega)$ (Fig. 1(d)) our theoretical curve displays a peak at $2.7 \mathrm{eV}$, that is noticeably higher than the experimentally observed 
peak. Here, however, the overestimation is surprising since we used an inverse life time of 0.06 Ry for iron, higher than the 0.05 Ry used by Ref. 15 .

Employing the KK transform method outlined earlier, we calculated $\sigma_{x x}^{2}(\omega)$ and $\sigma_{x y}^{1}(\omega)$. Figure 2(a) shows the result for $\omega \sigma_{x x}^{2}(\omega)$ in the case of nickel while Fig. 2(b) shows the one for iron. In both cases, our results are remarkably close to the results of Ref. 15. In the case of nickel they also compare well with experiments.53 In the case of iron the two experimental results seem to differ quite widely above $2 \mathrm{eV}$, thus making comparison with theory quite difficult. Figures 2(c) and Fig. 2(d) show our results for $\omega \sigma_{x y}^{1}(\omega)$. For nickel, the disagreement at $5 \mathrm{eV}$ is very obvious. In our calculations, this does not seem to have affected the polar Kerr angles as seriously as it has the equatorial Kerr effect in the 0-10 eV region. Other than this, the results for nickel seem to agree very well with the general features of the experiment. For iron, the results of $\omega \sigma_{x y}^{1}(\omega)$ follow the experiment quite well and also compare well with the results of Ref. 15.

\section{B. MOKE and X-MCD}

Using the calculated curves for the elements of the conductivity tensor we evaluated the Kerr angles for nickel and iron for the polar Kerr geometry using Eq. (11). The resulting curves for the optical region are shown in Fig. 3(a) and Fig. 3(b). In the calculation of the Kerr angles, we have included the effect of a phenomenological Drude term using values for $\sigma_{D}$ and $\tau_{D}$ from previous experimental results. 5 The results on nickel are particularly good since the shift of $1 \mathrm{eV}$ is not as noticeable in the Kerr angle spectra as it is in the elements of the conductivity tensor. This is in slight contrast to the observations of Ref. 15.

In a later publication 16 Oppeneer et al have investigated the dependence of MOKE spectra on the strength of the spin-orbit coupling. They concluded that the MOKE peaks scale linearly with the spin orbit coupling parameter $\xi$ and varying $\xi$ could produce a better agreement with the observed MOKE spectra for nickel. No such adjustments for $\xi$ were necessary in our results and our theoretical results for nickel seem to agree very well with 
the experimental results of Ref. 55 throughout the energy range of the data. In the light of these observations we may conclude that the spin-orbit coupling strength in our $d$-bands is quite accurate. In the case of iron, our Kerr angles are in excellent agreement with experimental results 55 and also compare well with the theoretical results of Ref. 15 and Ref. 18 .

As noted before, one of the features of our KK transformation method is that for functions that do not decay to zero within the required energy range of interest, accurate momentum matrix elements for energies up to two times as much are needed to correctly produce the KK transformation. Our momentum matrix elements are sufficiently accurate up to those energies since they are calculated using simple analytic expressions resulting from the use of a Gaussian basis and hence are free of any numerical approximations. In addition, it is evident from our results of Kerr angles that with a straightforward inclusion of spin-orbit coupling (for which we again have analytic expressions) in a manner described in the earlier section, we are able to very effectively and efficiently account for all the principal features of the MOKE spectra.

It is interesting to see whether the strength of the spin-orbit coupling in our $d$-bands that has given us good results for MOKE, gives consistent results for the other magnetooptical properties as well. This is a very important test since it is now widely accepted 15,18 that MOKE depends sensitively on the strength of the spin-orbit coupling and the exchange splitting. To further investigate whether or not our spin-orbit coupling in the valence bands is accurate we decided to calculate the X-ray MCD spectra of iron and nickel at the $2 p$ edge using our first principles LCGO method. This serves as a simultaneous check for the accuracy of the LDA at X-ray energies. In the X-MCD at the $2 p$ core edge, it is known that while the separation between the two peaks originates principally from the spin-orbit splitting of the $2 p$ levels, the exact ratio between the two peaks (e.g. approximately -1.6:1 in the case of nickel) arises out of the spin-orbit splitting of the $3 d$ valence bands. Since XMCD is the difference in absorption of right and left circularly polarized X-rays in the polar 
geometry, this is nothing but $\sigma_{x y}^{2}(\omega)$ evaluated using momentum matrix elements between core $2 p$ and valence $3 d$ bands.

The results of this calculation are shown in Fig. 4(a) for nickel and Fig. 4(b) for iron. Along with this, we also computed the $\sigma_{x x}^{1}(\omega)$ which is a measure of the total absorption of right and left circularly polarized X-rays. These results are shown in Fig. 5(a) and Fig. 5(b). As in any core to band transition the effect of the core hole has to be accounted for, which we did by recalculating bands and eigenfunctions with an increased effective $Z$ value and then using the new energies and wavefunctions for the final state to calculate the momentum matrix elements. The resulting $\mathrm{L}_{3}$ and $\mathrm{L}_{2}$ peaks occur at $856 \mathrm{eV}$ and $874 \mathrm{eV}$ for nickel, which agree fairly well with the observed energies of $853 \mathrm{eV}$ and $871 \mathrm{eV}$ respectively. Similarly, the calculated $\mathrm{L}_{3}$ and $\mathrm{L}_{2}$ peaks for iron occur at $710 \mathrm{eV}$ and $723 \mathrm{eV}$, which agree fairly well with the observed peaks of $707 \mathrm{eV}$ and $720 \mathrm{eV}$ respectively. However, our main results in the X-MCD spectra are the $\mathrm{L}_{3}$-to- $\mathrm{L}_{2}$ ratios which are $-1.56: 1$ in the case of nickel and $-1.2: 1$ in the case of iron. For nickel this seems to agree very well with the observed ratio of -1.6:1. For iron the theoretical ratio is slightly smaller than the observed ratio. However it agrees with the ratio for iron determined by other first principle methods based on LDA.22.24 Thus, the discrepancy in the $\mathrm{L}_{3}$-to- $\mathrm{L}_{2}$ ratio for iron is not an artifact of our method but may be due to the failure of the one-electron band picture. One aspect that must be pointed out in the X-MCD spectra of nickel is the missing peaks, often referred to as $\mathrm{B}$ and $\mathrm{B}^{\prime}, 4 \mathrm{eV}$ away from the principal peaks on the high energy side. These have been ascribed to many body effects in nickel by past author\$56 and have been reproduced by Jo and Sawatzky in a many-body calculation on nickel.5-5 They have not been reproducible by any one-electron band calculation. The results of our band-structure based calculation of MOKE and X-MCD are then an indication that, within the LDA framework, our method does give an accurate and consistent description of these phenomena. 


\section{The Equatorial Kerr effect and Photoabsorption X-MLD}

The other principal tool for magneto-optical studies on ferromagnetic materials has been

the equatorial Kerr effect 28,58 As mentioned above, the reflection coefficient of an incident electromagnetic wave in this geometry depends on the sense of magnetization in the metal. When observed at a core-level edge this phenomenon may be termed X-ray magnetic linear dichroism. It may be noted that a different kind of absorption X-MLD can be obtained by keeping the magnetization constant but rotating the photon polarization vector by $90^{\circ}$. This effect has also been calculated previously22 but is not considered here.

We have calculated the equatorial Kerr effect for absorption both in the optical region and in the soft X-ray region (X-MLD) using Eq. (12) for several different angles of incidence. The results for the optical region are shown Fig. 6(a) and Fig. 6(b) for nickel and iron respectively. From Fig. 6(a) it can be seen that as the angle of incidence is changed from $45^{\circ}$ to $80^{\circ}$ on nickel the sign of the effect is reversed. After this, from $80^{\circ}$ to $88^{\circ}$ the peak magnitudes progressively increase reaching a peak somewhere close to grazing incidence. The effect, of course, disappears at exactly $90^{\circ}$. For nickel, it is important to remember that in the evaluation of the conductivity tensor, the theoretical results have always predicted a dip at $5 \mathrm{eV}$ where there actually is an experimental rise (see Fig. 1(b) and Fig. 1(d)). Taking a clue from this observation, we may predict that in Fig. 6(a), although the structure up to $4 \mathrm{eV}$ may compare well with experiment, the peaks (or dips) at about $5 \mathrm{eV}$ may well be found to be reversed for every angle.

The curves of Fig. 6(b) for iron can however be considered to be faithfully reproducing experimental results. For iron, we see that as the angle is increased from $80^{\circ}$ to $85^{\circ}$, there is a reversal of the sign of the effect only for the region upto $5 \mathrm{eV}$. The effect is most enhanced at $88^{\circ}$ to $89^{\circ}$ and the most observable change is at about $6 \mathrm{eV}$. Although we did not find any experimental results for change in absorption upon reversal of magnetization in the optical regime, experimental results for the change in the reflection intensity between magnetized and unmagnetized nickel have been reported in the past 28 We calculated this also using 
Eq. (12) by putting $\sigma_{x y}$ equal to zero to simulate the unmagnetized nickel. Although this procedure may be viewed with caution, our results for nickel as shown in Fig. 6(c) agree remarkably well with experiment. There again is the characteristic dip at $5 \mathrm{eV}$ which does not follow the rise seen in experiment, but this has already been noted to be a consistent failure of LDA in nickel.

For the X-ray region our results for the $2 p$ edge have been shown for nickel and iron in Fig. 7(a) and Fig. 7(b) respectively. We clearly see that at the onset of the $\mathrm{L}_{2,3}$ edges in both cases the sign of the peaks is the same. There is, however, a sharp overshoot to the opposite sign just after the $\mathrm{L}_{2}$ edge. This is more pronounced in the case of nickel, although it is unmistakable even in iron. It would be interesting to see if these features are actually observed. In the case of iron, the predicted peaks at the $\mathrm{L}_{3}$ and $\mathrm{L}_{2}$ edge seem to be of almost equal magnitude. This may be in slight disagreement with experimental results since, as mentioned earlier, the prediction of the $\mathrm{L}_{3}$-to- $\mathrm{L}_{2}$ ratios both in the $\mathrm{X}-\mathrm{MCD}$ and total X-ray absorption calculation are slightly underestimated for iron. It may also be noted that at the incident angles considered the maximum effect for iron is about $0.8 \%$ while that for nickel is about 1\%. This agrees with the analysis about this type of linear dichroism done in the past.22 To the best of our knowledge, such absorption X-MLD experiments at the $2 p$ edge for iron and nickel have not been reported although X-MLD in photoemission has been subjected to an onslaught of theoretical and experimental analysis. 11, 12,59, 62 The results there are characteristically different from our absorption results, since the final states in a photoemission experiment are very high above the $3 d$ bands. As such, the final state spin-polarization does not play as significant a role as it does in photoabsorption type measurements. This is particularly demonstrated in photoemission-XMLD62 where the signs of the two peaks are opposite each other, in contrast to what is seen in our photoabsorptionX-MLD. 


\section{Soft X-ray Faraday rotation}

We finally turn to the Faraday rotation of the plane of polarization of linearly polarized X-rays upon transmission through magnetic metals. The main concern here stems from the knowledge that the Eq. (13) derived for the Faraday effect is based on the dipole approximation. At a first glance, to use it for evaluating the Faraday effect in the soft-X-ray regime is to bring the approximation to a questionable limit. This problem has been addressed previously27 and it has been demonstrated that the theory of Faraday effects can be extended to the X-ray regime by postulating the existence of a free carrier effective dielectric medium with the same conductivity tensor as the ferromagnetic metal in question. In fact we already did make this extension when we used $\sigma_{x y}^{2}$ to determine X-MCD and $\sigma_{x x}^{1}$ to determine the total absorption. Since those results produced reasonably good agreement with experiments, we wish to examine its applicabilty further by evaluating the Faraday effect at soft X-ray energies. This calculation is a trivial extension to the calculation of X-MCD and total absorption since all we need is the KK tranforms of these curves. This, as mentioned earlier, can be done very efficiently and accurately with our method.

The calculated Faraday rotation at the $2 p$ edges of nickel and iron is exhibited in Fig. 8(a) and Fig. 8(b). An experiment on soft X-ray Faraday rotation has recently been done at the $2 p$ edge of iront with which we compare our results. As can be seen from Fig. 7(b) the agreement with experiment is rather good at the $\mathrm{L}_{2}$ edge but is relatively poor at the $\mathrm{L}_{3}$ edge. This again is related to the underestimated peak in the MCD and total absorption spectra at the $\mathrm{L}_{3}$ edge of iron. Nevertheless, given that the dipole approximation is not strictly valid at these energies, the agreement with observed angles can be taken to be quite good. For the case of nickel, because our X-MCD and total absorption results are in much better agreement with experiments, we are more confident of the relative magnitudes of angles at the $\mathrm{L}_{3}$ and $\mathrm{L}_{2}$ edges. 


\section{CONCLUSIONS}

A first principles, self consistent LCGO band calculation has been performed and the principal magneto-optical properties have been computed for bulk iron and nickel in this paper. In spite of the simplistic way in which spin-orbit coupling has been included, our results have agreed very well with previous first principles calculations based on LDA of MOKE and MCD and experimental observations. In particular our MOKE results for nickel have produced very good agreement with experiments, despite the lack of good description of some of the LDA bands in nickel. Discrepancies originating from the failure of the LDA have however crept up in the equatorial Kerr effect in the optical region around $5 \mathrm{eV}$. Our results for photoabsorption X-MLD could not be compared with experiments but judging from the agreement of the X-MCD and soft X-ray rotation results with experiments, we conclude that our results for nickel may be considered to be accurate, but for iron the actual $\mathrm{L}_{3}$ peak magnitude may be much higher as compared to the $\mathrm{L}_{2}$ peak. This conclusion is also supported by our Faraday effect results on iron. It is also noteworthy that the equatorial Kerr effect is larger in the optical region than in the soft-X-ray region.

We have also demonstrated that fast, efficient and accurate KK transforms can in fact give sufficiently satisfactory results for sensitive magneto-optical effects of bulk metals, which have been previously obtained by computationally intensive methods. This speed and efficiency is particularly important if one is to use such calculations for quickly obtainable results for technological applications. This analysis has also confirmed that expressions for the Faraday effect for the optical region may be extended to the soft X-ray region yielding satisfactory results. The principal feature of this work is however that a single calculation of all the components of the conductivity tensor in both the optical as well as the X-ray region has yielded consistent results for a host of different magneto-optical effects. 


\section{ACKNOWLEDGMENTS}

One of us (N.M) would like to acknowledge K. Subramanian for useful discussions in the

theory of the equatorial Kerr effect. This research was supported by the National Science Foundation under Grant Nos. NSF-DMR-9120166 and NSF-DMR-9408634. 


\section{REFERENCES}

${ }^{1}$ W. Voigt, Magneto- und Electrooptik. Leipzig: Teubner, (1908).

${ }^{2}$ W. Schlichting, T. D. Milster, M. S. Wang, and C. Brucker, J. Appl. Phys. 75, 2322 (1994)

${ }^{3}$ J. Stöhr, Y. Wu, B. D. Hermsmeier, M. G. Samant, G. R. Harp, S. Koranda, D. Dunham, and B. P. Tonner, Science 259, 658 (1993).

${ }^{4}$ W. H. Meiklejohn, Proc. IEEE 74, 1570 (1986).

${ }^{5}$ M. H. Kryder, J. Appl. Phys. 57, 3913 (1985).

${ }^{6}$ H. R. Zhai, S. M. Zhou, M. Lu, Y. Z. Miao, B. X. Gu, S. L. Zhang, H. Wang, and H. B. Huang, J. Magn. Magn. Mater. 104-107 1015 (1992).

${ }^{7}$ J. B. Kortright, M. Rice, and R. Carr, Phys. Rev. B 51, 10240 (1995).

${ }^{8}$ G. Schütz, W. Wagner, W. Wilhelm, P. Kienle, R. Zeller, R. Frahm, and G. Materlik, Phys. Rev. Lett. 58, 737 (1987)

${ }^{9}$ C. T. Chen, F. Sette, Y. Ma, and S. Modesti, Phys. Rev. B 42, 7262 (1990).

${ }^{10}$ L. Baumgarten, C. M. Schneider, H. Petersen, F. Schäfers, and J. Kirschner, Phys. Rev. Lett. 65, 492 (1990).

${ }^{11}$ Gerrit van der Laan, Phys. Rev. B 51, 240 (1995).

12 J. Henk, S. V. Halihov, T. Scheunemann, and R. Feder, Phys. Rev. B 50, 8130 (1994).

${ }^{13}$ H. S. Bennet and E. A. Stern, Phys. Rev. 137, A448 (1965)

${ }^{14}$ J. L. Erskine and E. A. Stern, Phys. Rev. B 12, 5016 (1975)

${ }^{15}$ P. M. Oppeneer, T. Maurer, J. Sticht, and J. Kübler, Phys. Rev. B 45, 10924 (1992).

${ }^{16}$ P. M. Oppeneer, J. Sticht, T. Maurer, and J. Kübler, Z. Phys. B 88, 309 (1992).

${ }^{17}$ T. Maurer, J. Sticht, P. M. Oppeneer, F. Herman, and J. Kübler, J. Magn. Magn. Mater. 
104-107, 1029 (1992).

${ }^{18}$ G. Y. Guo and H. Ebert, Phys. Rev. B 50, 10377 (1994).

${ }^{19}$ G. Y. Guo and H. Ebert, Phys. Rev. B 51, 12633 (1995).

${ }^{20}$ H. Ebert, P. Strange, and B. L. Gyorffy, J. Appl. Phys. 63, 3055 (1988).

${ }^{21}$ H. Ebert and R. Zeller, Phys. Rev. B 42, 2744 (1990).

${ }^{22}$ G. Y. Guo, H. Ebert, W. M. Temmerman, and P. J. Durham, Phys. Rev. B 50, 3861 (1994).

${ }^{23}$ R. Wu, D. Wang, and A. J. Freeman, Phys. Rev. Lett. 71, 3581 (1993).

${ }^{24}$ R. Wu and A. J. Freeman, Phys. Rev. Lett. 73, 1994 (1994).

25 J. Igarashi and K. Hirai, Phys. Rev. B 50, 17820 (1994).

${ }^{26}$ G. Y. Guo, H. Ebert, W. M. Temmermann, and P. J. Durham, in Metallic Alloys: Experimental and Theoretical Prespectives, edited by J. S. Faulkner and R. G. Jordan (Kluwer Academic, Dordrecht, 1994)

${ }^{27}$ H. J. Gotsis and P. Strange, J. Phys. Condens. Matter 6, 1409 (1994).

${ }^{28}$ G. S. Krinchik and A. A. Gorbacher, Phys. Metals Mettalog. 11, 49 (1961).

${ }^{29}$ C. S. Wang and J. Callaway, Phys. Rev. B 9, 4897 (1974).

${ }^{30}$ M. Singh, C. S. Wang, and J. Callaway, Phys. Rev. B 11, 287 (1975).

${ }^{31}$ C. S. Wang and J. Callaway, Comput. Phys. Commun. 14, 327 (1978).

${ }^{32}$ A. K. Rajagopal, S. P. Singhal, and J. Kimball (unpublished), as quoted by A. K. Rajagopal in Advances in Chemical Physics, edited by G. I. Prigogine and S. A. Rice (Wiley, New York, 1979), Vol.41, p.59.

33 J. Rath and A. J. Freeman, Phys. Rev. B 11, 2109 (1975). 
34 S. P. Singhal, Phys. Rev. B 12, 564 (1975).

${ }^{35}$ P. Blaha and J. Callaway, Phys. Rev. B 32, 7664 (1985).

${ }^{36}$ J. Callaway and C. S. Wang, Phys. Rev. B 16, 2095 (1977).

${ }^{37}$ C. S. Wang and J. Callaway, Phys. Rev. B 15, 298 (1977).

${ }^{38}$ D. G. Laurent, C. S. Wang, and J. Callaway, Phys. Rev. B 17, 455 (1978).

${ }^{39}$ D. G. Laurent, J. Callaway, and C. S. Wang, Phys. Rev. B 20, 1134 (1979).

${ }^{40}$ D. G. Laurent, J. Callaway, J. L. Fry, and N. E. Brener, Phys. Rev. B 23, 4977 (1981).

${ }^{41}$ A. R. Jani, N. E. Brener, and J. Callaway, Phys. Rev. B 38, 9425 (1988).

${ }^{42}$ G. S. Tripathi, N. E. Brener, and J. Callaway, Phys. Rev. B 38, 10454 (1988).

${ }^{43}$ H. Chen, N. E. Brener, and J. Callaway, Phys. Rev. B 40, 1443 (1989).

${ }^{44}$ A. R. Jani, G. S. Tripathi, N. E. Brener, and J. Callaway, Phys. Rev. B 40, 1593 (1989).

${ }^{45}$ A. J. H. Wachters, J. Chem. Phys. 52, 1033 (1970).

${ }^{46}$ J. E. Bertie and S. L. Zhang, Can. J. Chem. 70, 520 (1992).

${ }^{47}$ W. Reim, J. Magn. Magn. Mater. 58, 1 (1986).

${ }^{48}$ M. J. Freiser, IEEE Trans. Magn. MAG-4, 152 (1968).

49 J. M. Florczak and E. D. Dahlberg, Phys. Rev. B 44, 9338 (1991).

${ }^{50}$ H. Ehrenreich, H. R. Philipp, and D. J. Olechna, Phys. Rev. 131, 2469 (1963).

${ }^{51}$ A. Leibsch, Phys. Rev. Lett. 43, 1431 (1979).

${ }^{52}$ J. H. Weaver, E. Colavita, D. W. Lynch, and R. Rosei (unpublished).

${ }^{53}$ P. B. Johnson and R. W. Christy, Phys. Rev. B 9, 5056 (1974).

${ }^{54}$ A. P. Lenham and D. M. Treherne, in Optical Properties and Electronic Structure of Metals 
and Alloys (North-Holland, Amsterdam,1966), p.196.

${ }^{55}$ G. S. Krinchik and V. A. Artem'ev, Sov. Phys. JETP 26, 1080 (1968).

${ }^{56}$ N. V. Smith, C. T. Chen, F. Sette, and L. F. Mattheiss Phys. Rev. B 46, 1023 (1992).

${ }^{57}$ T. Jo and G. A. Sawatzky, Phys. Rev. B 43, 8771 (1991).

${ }^{58}$ M. E. Brubaker, E. R. Moog, C. H. Sowers, J. Zak, and S. D. Bader, J. Magn. Magn. Mater. 103, L7 (1992).

${ }^{59}$ F. Sirotti and G. Rossi, Phys. Rev. B 49, 15682 (1994).

${ }^{60}$ W. Kuch, M.-T. Lin, W. Steinhögl, C. M. Schneider, D. Venus, and J. Kirschner, Phys. Rev. B 51, 609 (1995).

${ }^{61}$ E. Tamura, G. D. Waddill, J. G. Tobin, and P. A. Sterne, Phys. Rev. Lett. 73, 1533 (1994).

${ }^{62}$ Ch. Roth, F. U. Hillebrecht, H. B. Rose, and E. Kisker, Phys. Rev. Lett. 70, 3479 (1993).

${ }^{63}$ M. Shiga and G. P. Pells, J. Phys. C 2, 1847 (1969).

${ }^{64}$ G. S. Krinchik and V. A. Artem'ev, J. Appl. Phys. 39, 1276 (1968).

${ }^{65}$ H. T. Yolken and J. Kruger, J. Opt. Soc. Am. 55, 892 (1965).

${ }^{66}$ P. G. van Engen, K. H. J. Buschow, and M. Erman, J. Magn. Magn. Mater. 30, 374 (1983). 


\section{FIGURES}

FIG. 1. Elements of the conductivity tensor for nickel and iron. In (a) and (b), solid line with an inverse lifetime of 0.0368 Ry is our result. Dashed line is the result of the calculation of Ref. 15 with an inverse lifetime of 0.04 Ry. In (c) and (d) solid line is our result for an inverse lifetime of 0.06 Ry. Dashed line is the result of the calculation of Ref. 15 for an inverse lifetime of 0.05 Ry. (a) $\sigma_{x x}^{1}$ for nickel. Circles are the experimental results of Ref. 50. Squares are the experimental results of Ref. 63. (b) $\omega \sigma_{x y}^{2}$ for nickel. Circles are the experimental results of Ref. 64. (c) $\sigma_{x x}^{1}$ for iron. Circles are the experimental results of Ref. 52. Squares are the experimental results of Ref. 65. (d) $\omega \sigma_{x y}^{2}$ for iron. Circles are the experimental results of Ref. 64.

FIG. 2. Dispersive components of the conductivity tensor for nickel and iron. (a) $\omega \sigma_{x x}^{2}$ for nickel. Circles are the experimental results of Ref. 53. (b) $\omega \sigma_{x x}^{2}$ for iron. Circles are the experimental results of Ref. 53. Squares are the experimental results of Ref. 65. (c) $\omega \sigma_{x y}^{1}$ for nickel. Circles are the experimental results of Ref. 64. (d) $\omega \sigma_{x y}^{1}$ for iron. Circles are the experimental results of Ref. 64.

FIG. 3. Polar Kerr rotation. In both graphs, solid line is our result. Dashed line is the result of the calculation of Ref. 15. (a) For nickel. Circles are the experimental results of Ref. 55. Squares are the experimental results of Ref. 66. (b) For iron. Circles are the experimental results of Ref. 55.

FIG. 4. X-MCD at the $2 p$ edges for (a) nickel and (b) iron. A Gaussian broadening of $0.2 \mathrm{eV}$ has been added in both cases.

FIG. 5. Total absorption at the $2 p$ edges for (a) nickel and (b) iron. A Gaussian broadening of $0.2 \mathrm{eV}$ has been added in both cases. 
FIG. 6. Equatorial Kerr effect. For (a) and (b) $\Delta T$ is the change in absorption intensity upon reversal in magnetization. $T$ is the average absorption of the two directions. Plots are correct upto a negative sign. (a) In absorption for nickel and (b) In absorption for iron. $\theta=45^{\circ}$ Solid line. $\theta=80^{\circ}$ Dotted line. $\theta=85^{\circ}$ Dashed line. $\theta=88^{\circ}$ Dash-dotted line. (c) $\theta=45^{\circ} . \Delta I$ is the change in reflection intensity upon magnetization. $I$ is the reflection intensity from unmagnetized nickel. Solid line is our theoretical result. Circles are the experimental results of Ref. 55 .

FIG. 7. Photoabsorption X-MLD at the $2 p$ edges. $\Delta T$ is the change in absorption intensity upon reversal in magnetization. $T$ is the average absorption of the two directions. Plots are correct upto a negative sign. (a) for nickel. $\theta=85^{\circ}$ Solid line. $\theta=87^{\circ}$ Dotted line. $\theta=88^{\circ}$ Dashed line. (b) for iron. $\theta=80^{\circ}$ Solid line. $\theta=85^{\circ}$ Dotted line. $\theta=87^{\circ}$ Dashed line.

FIG. 8. Soft X-ray Faraday rotation at the $2 p$ edges. In both figures solid line is our theoretical results. (a) for nickel. (b) for iron, $d=80 \mathrm{~nm}$. Circles are the experimental results of Ref. 7 . 


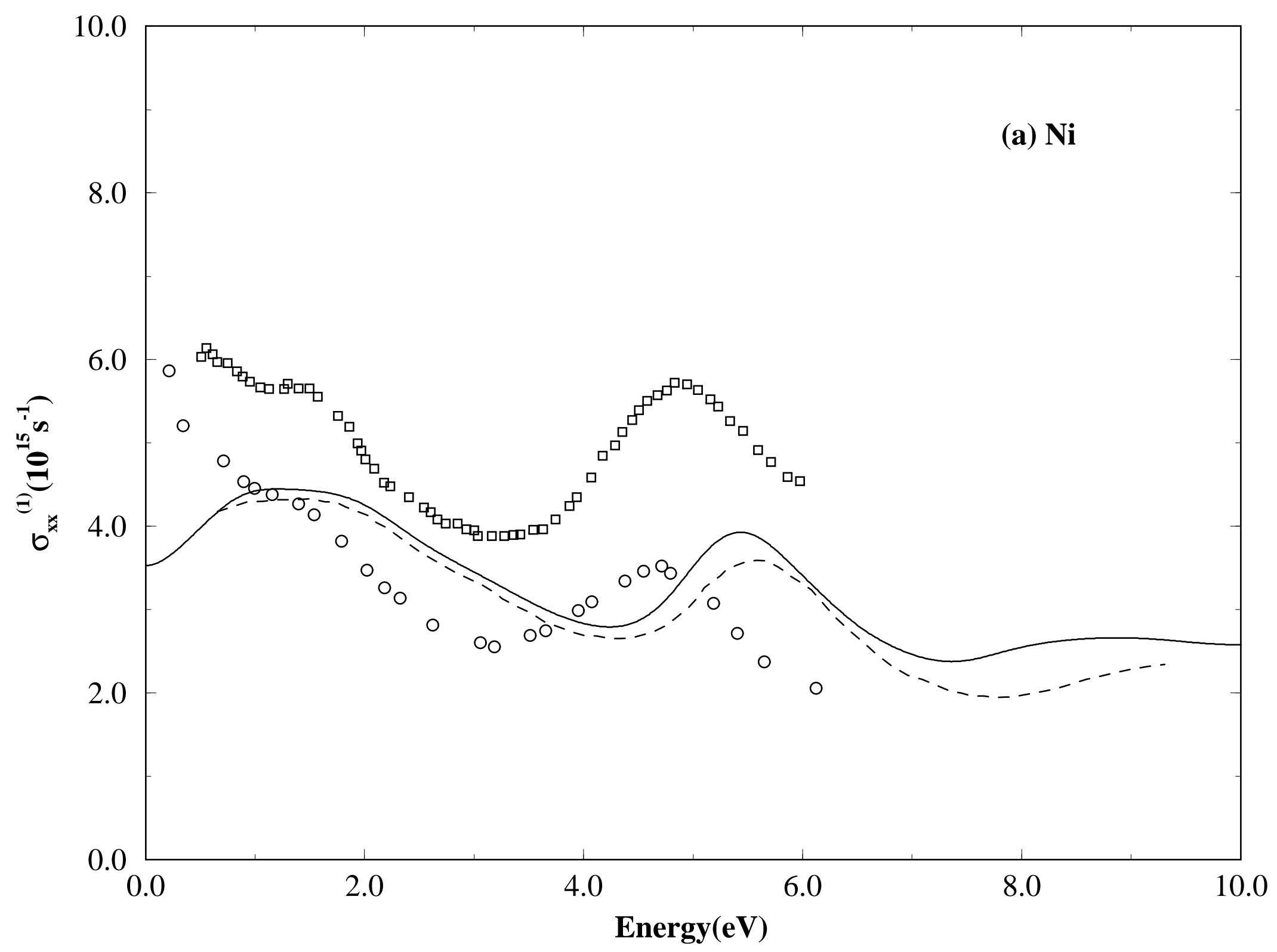




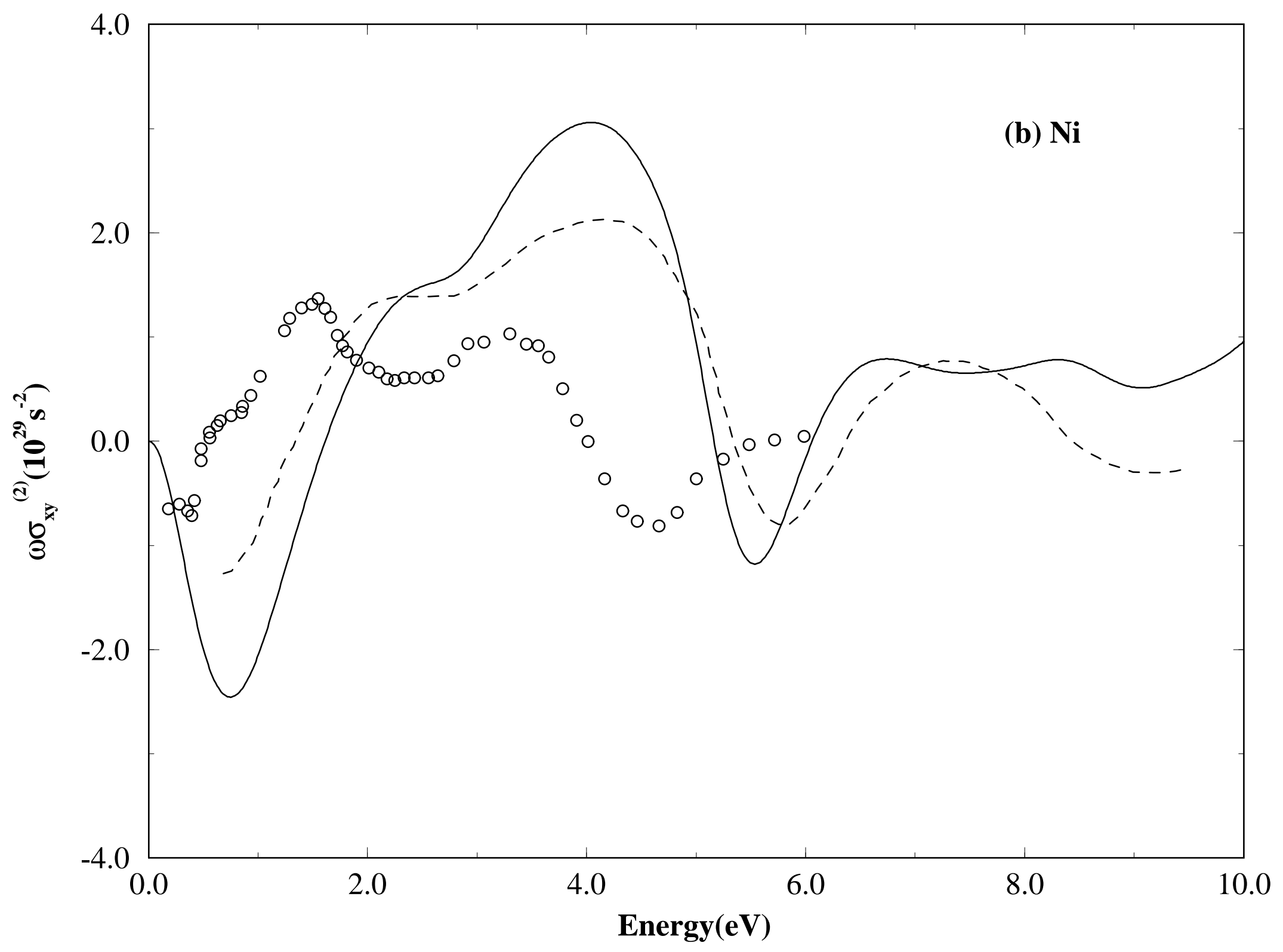




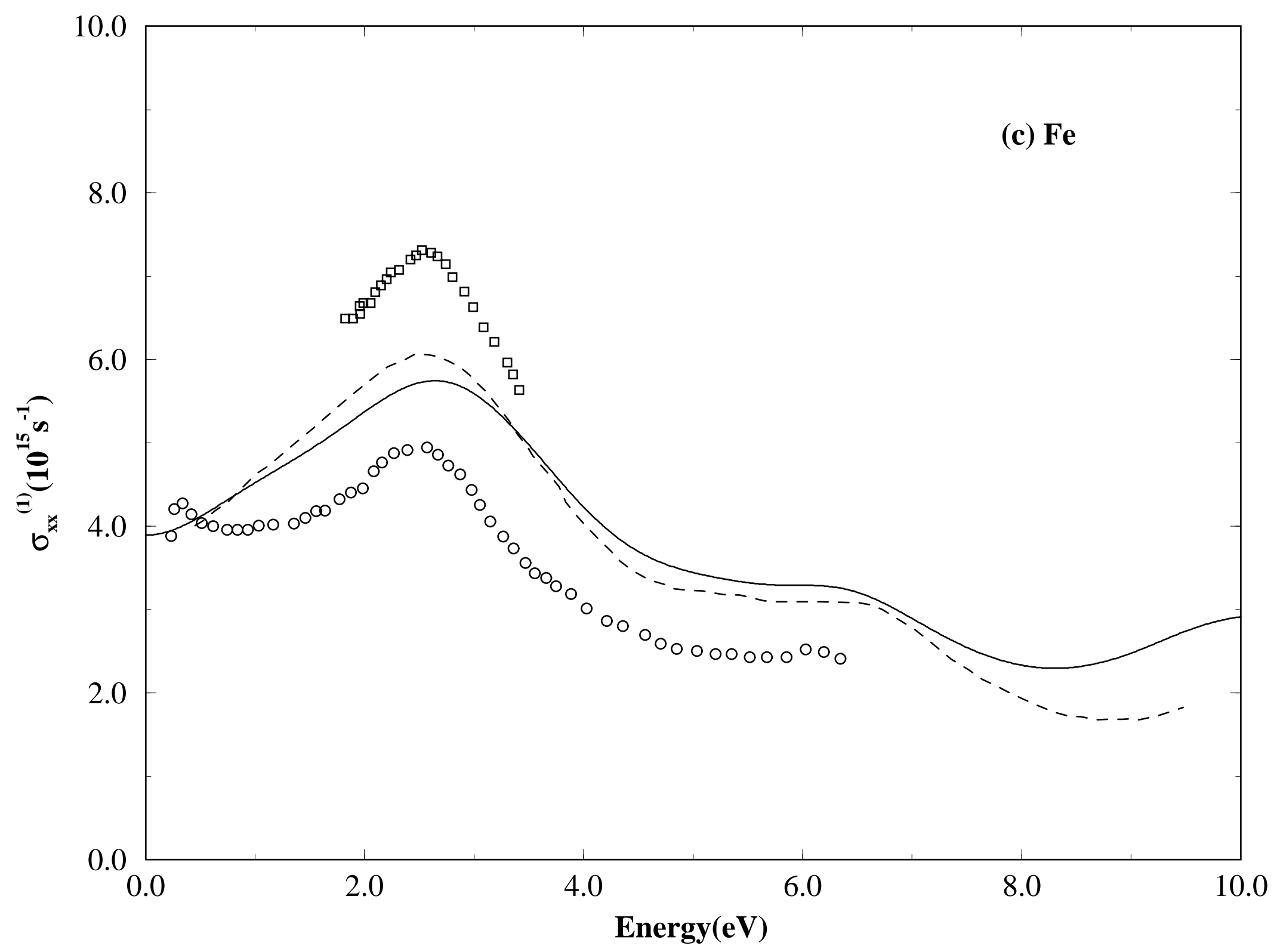




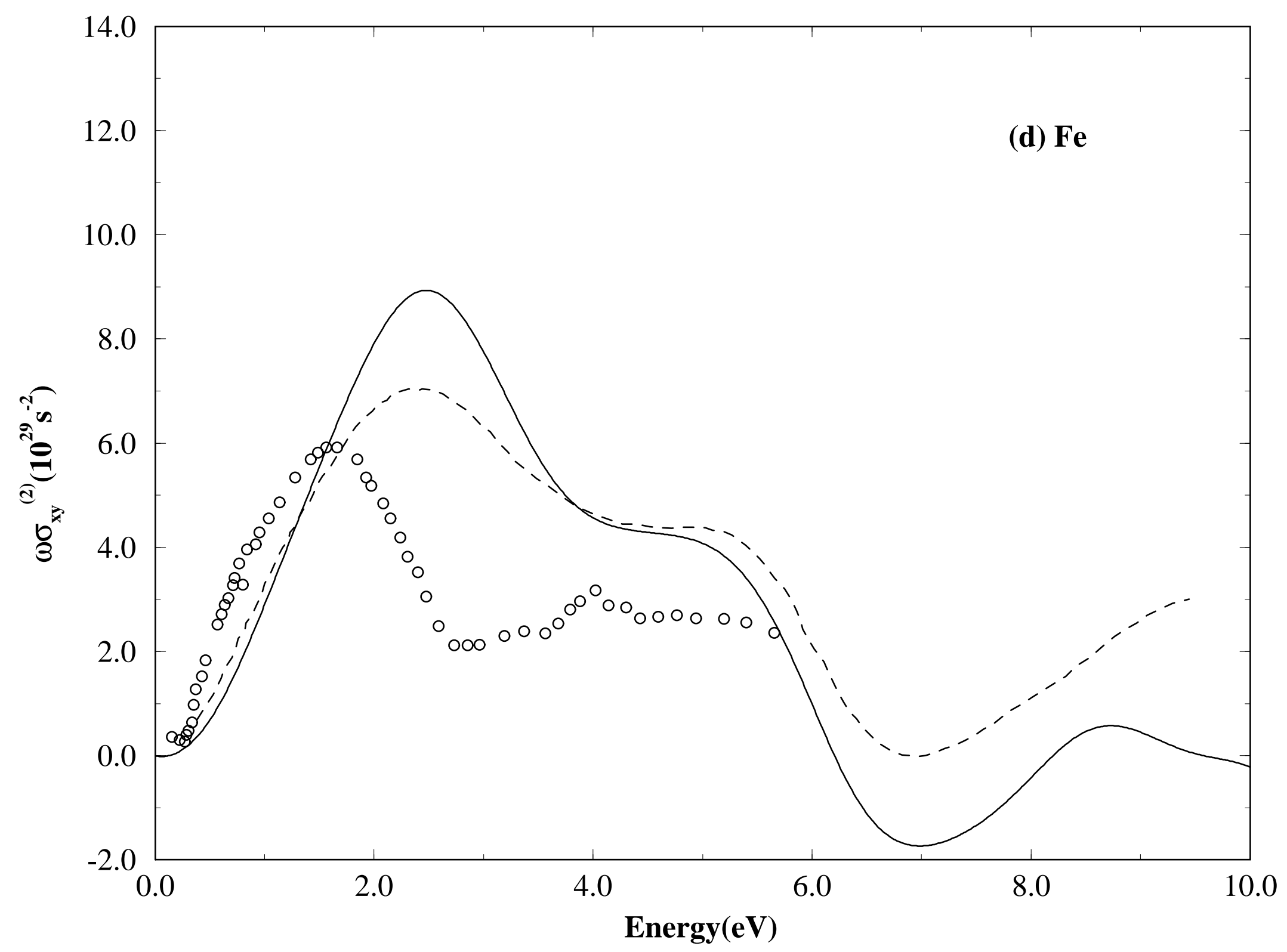




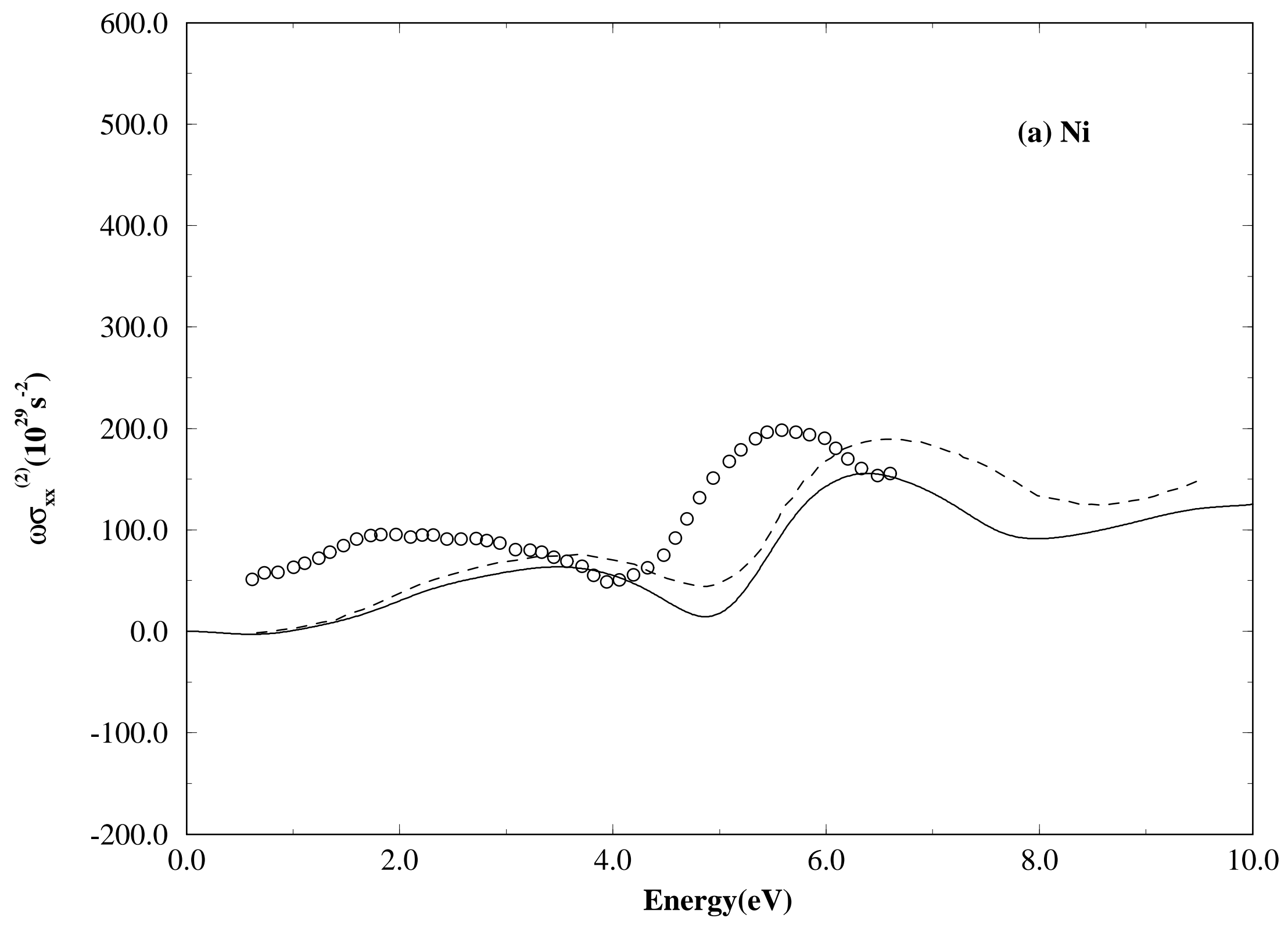




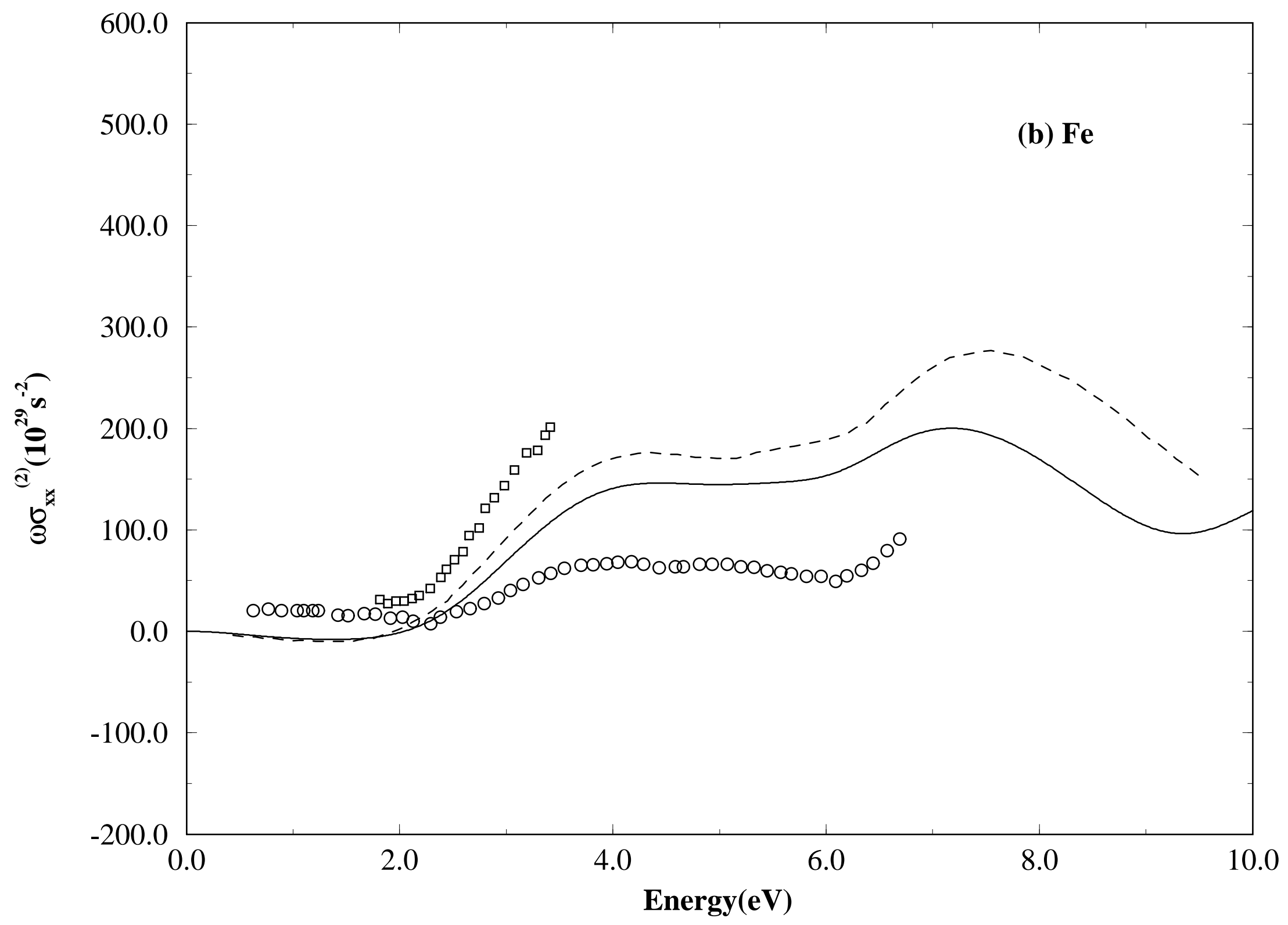




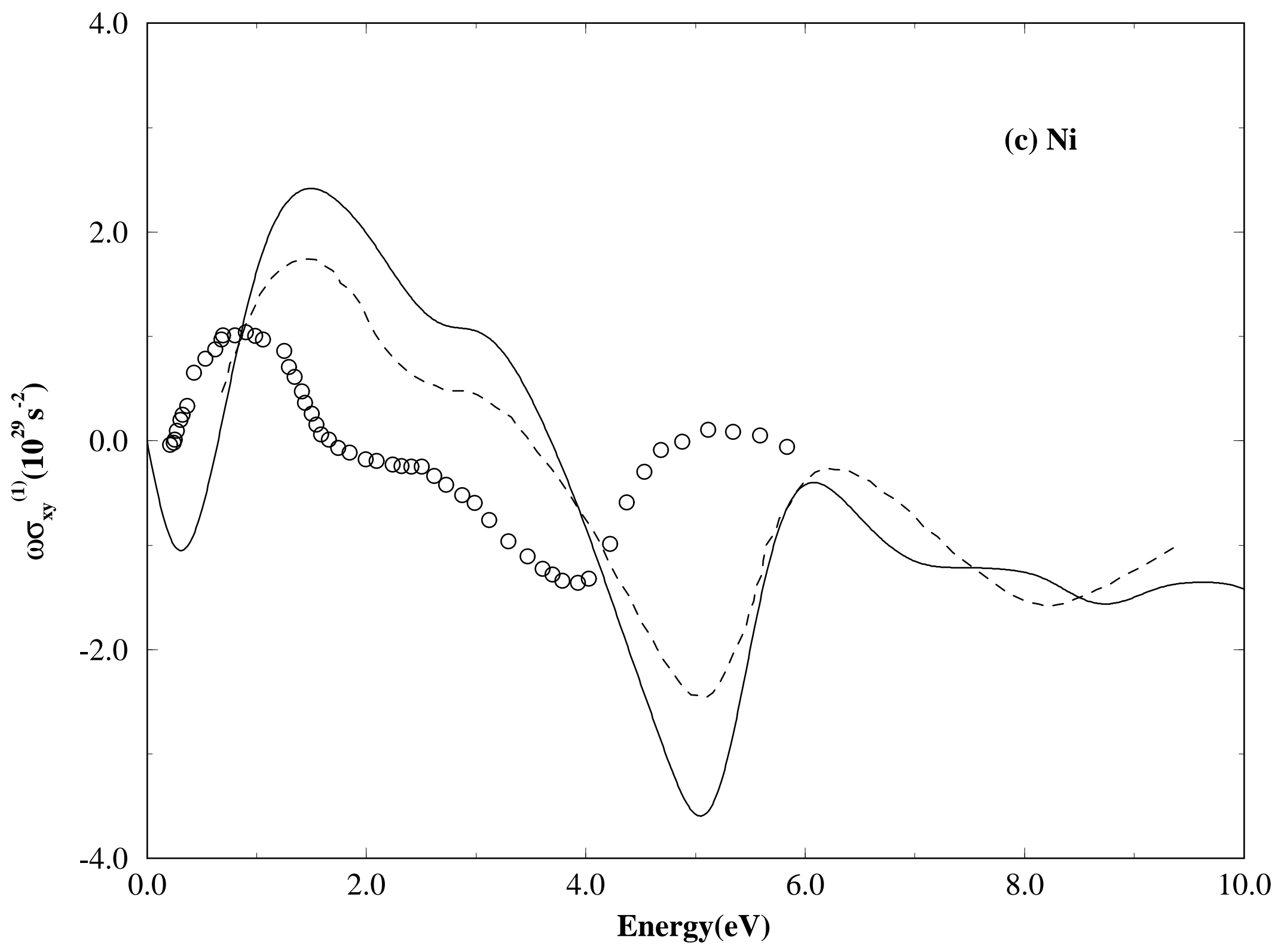




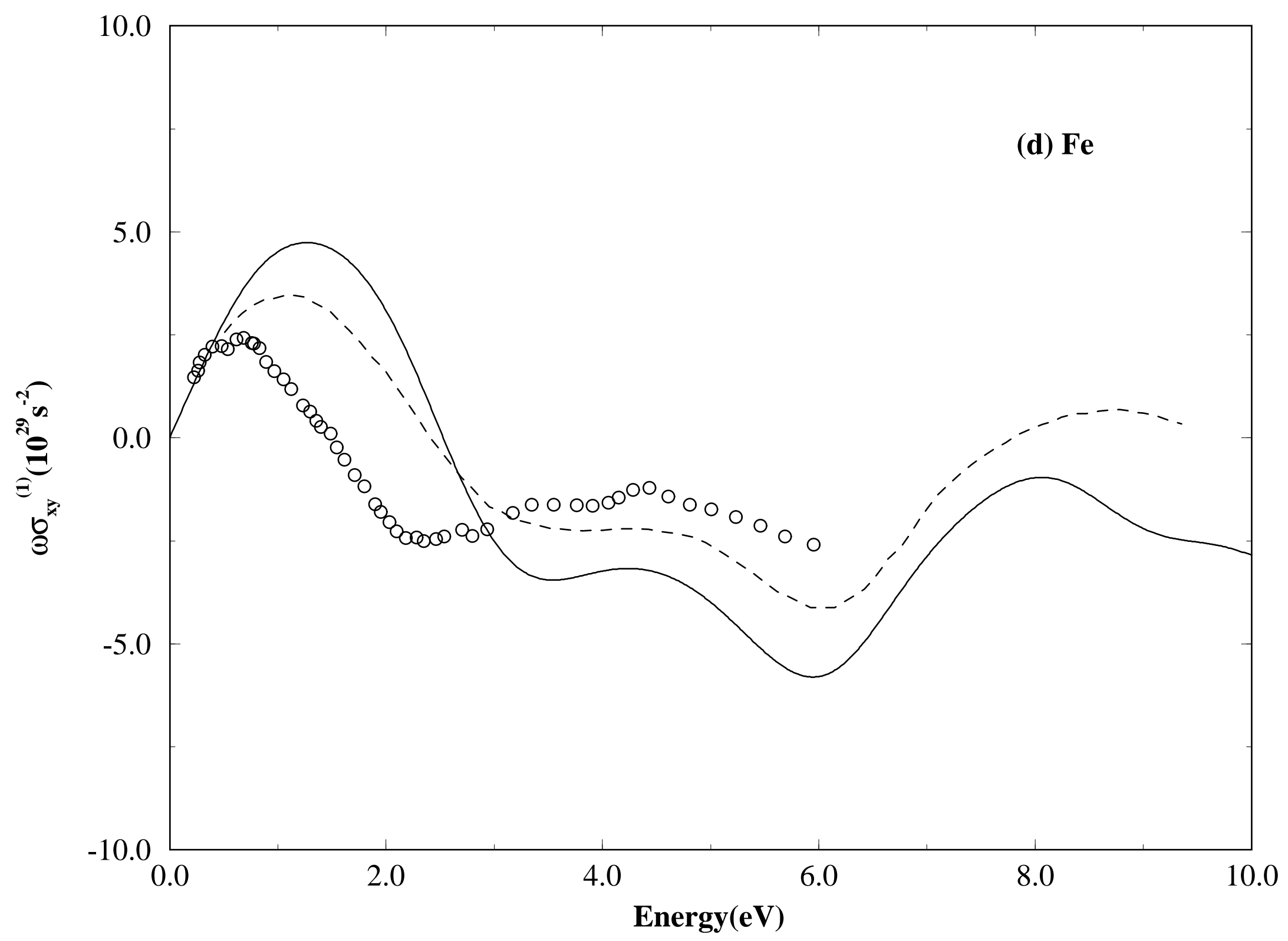




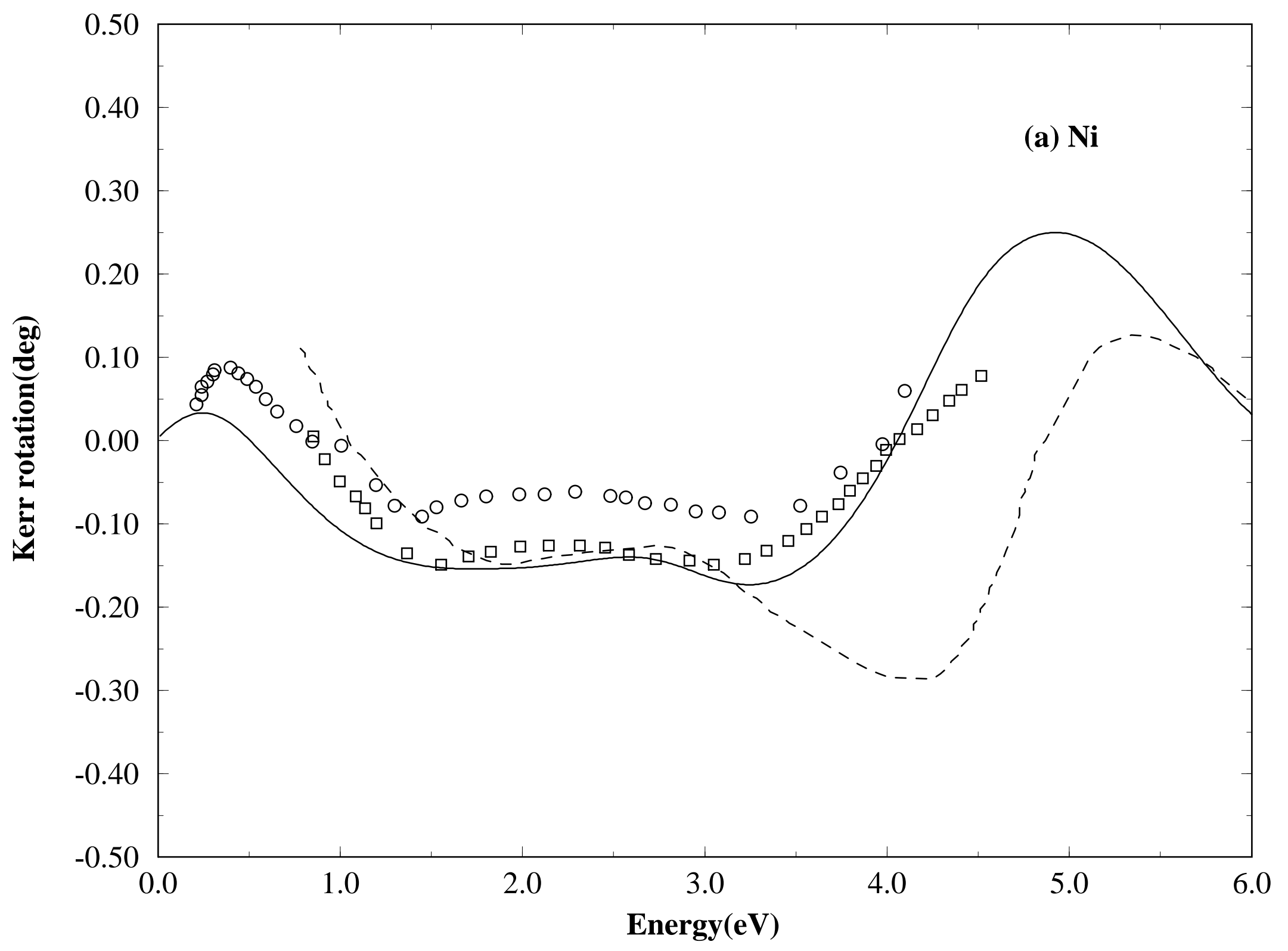




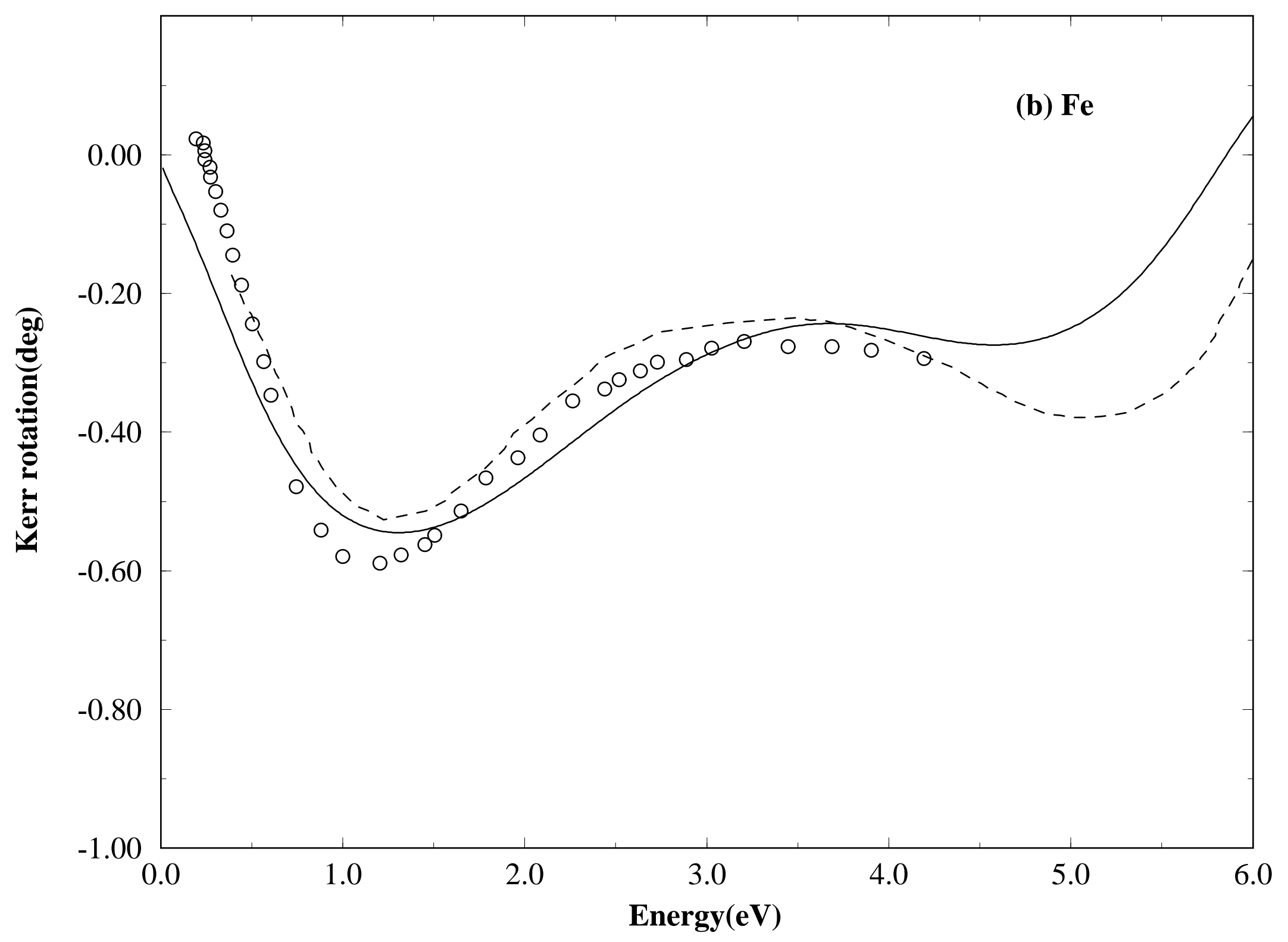




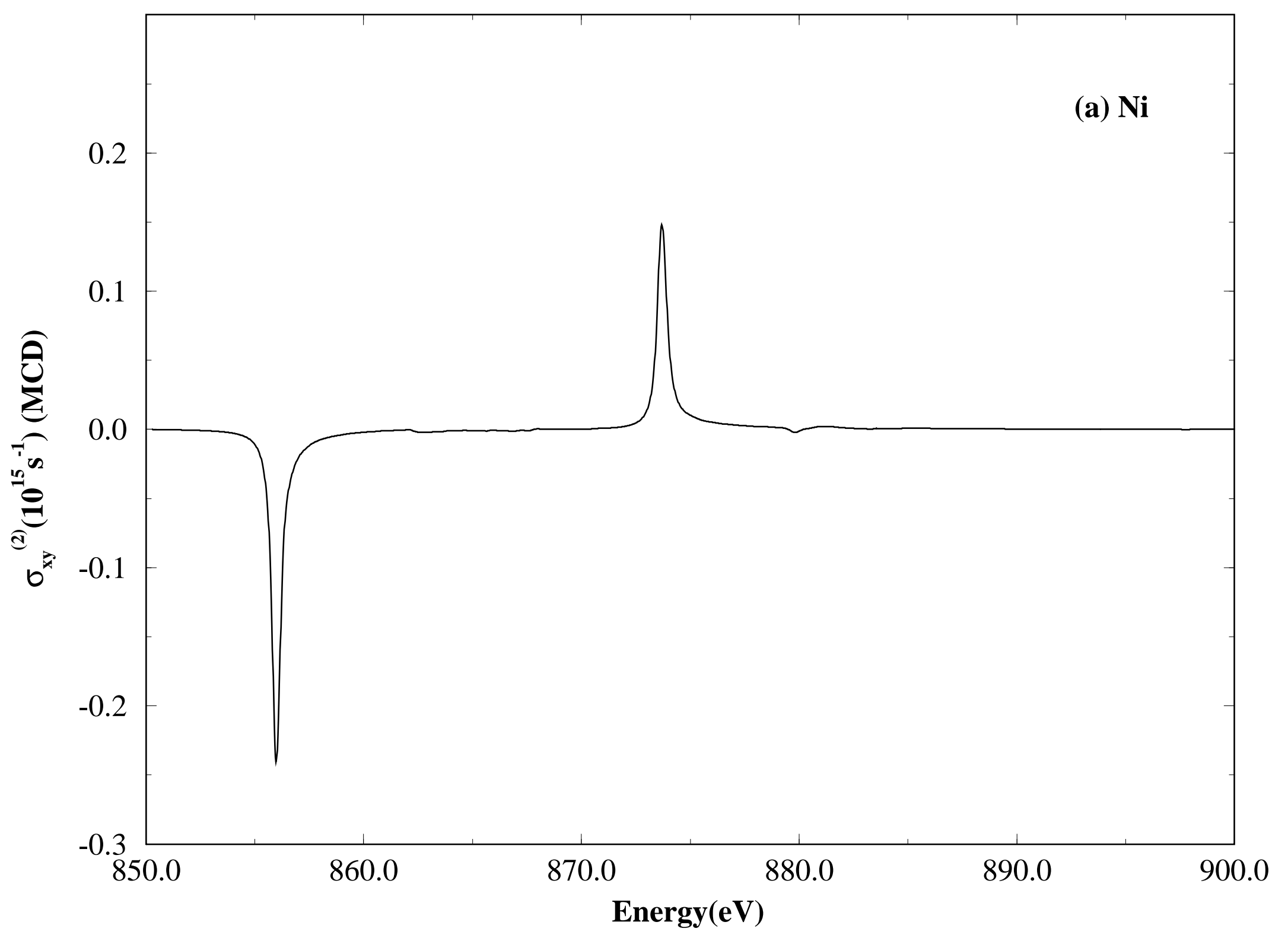




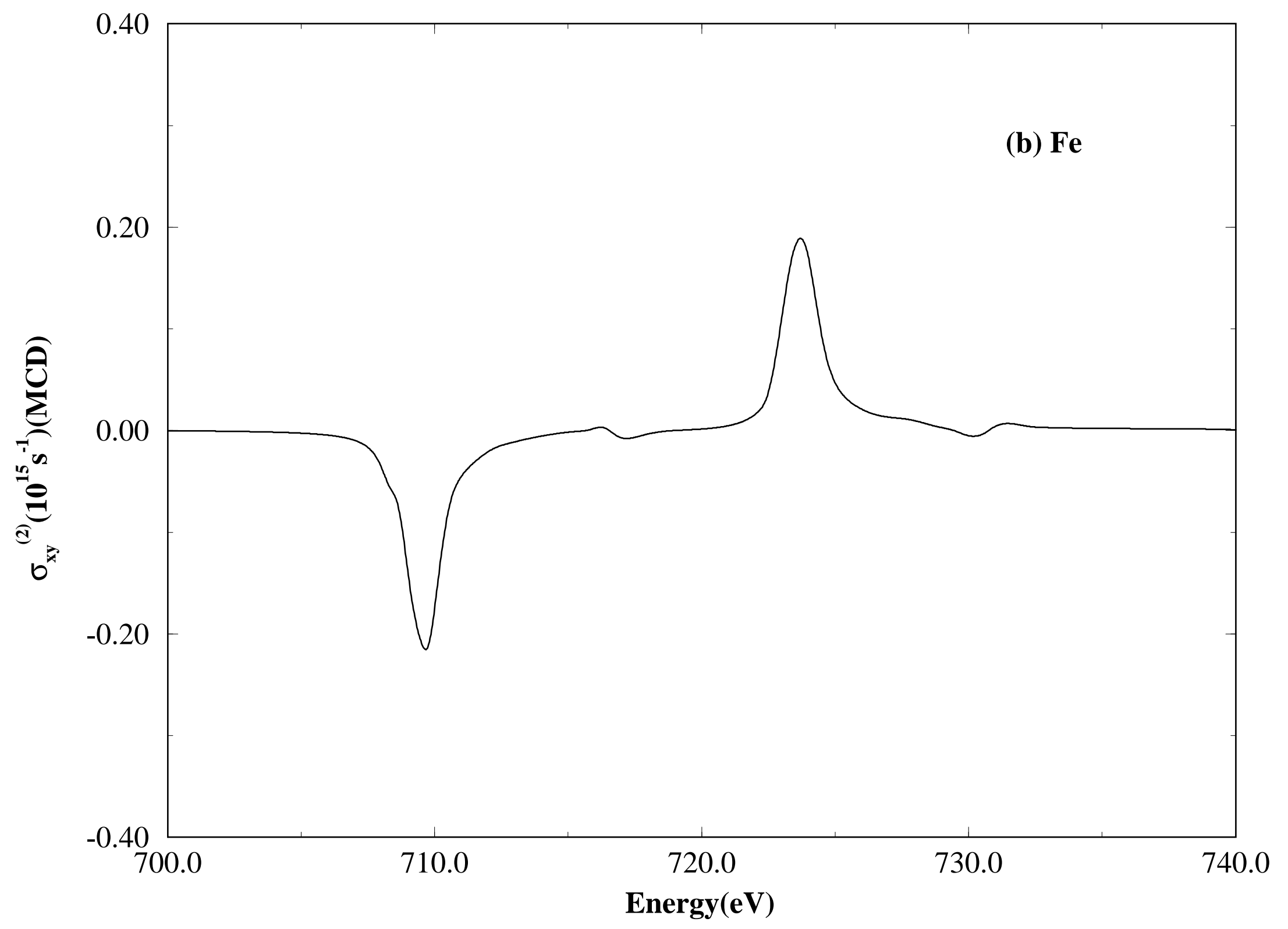




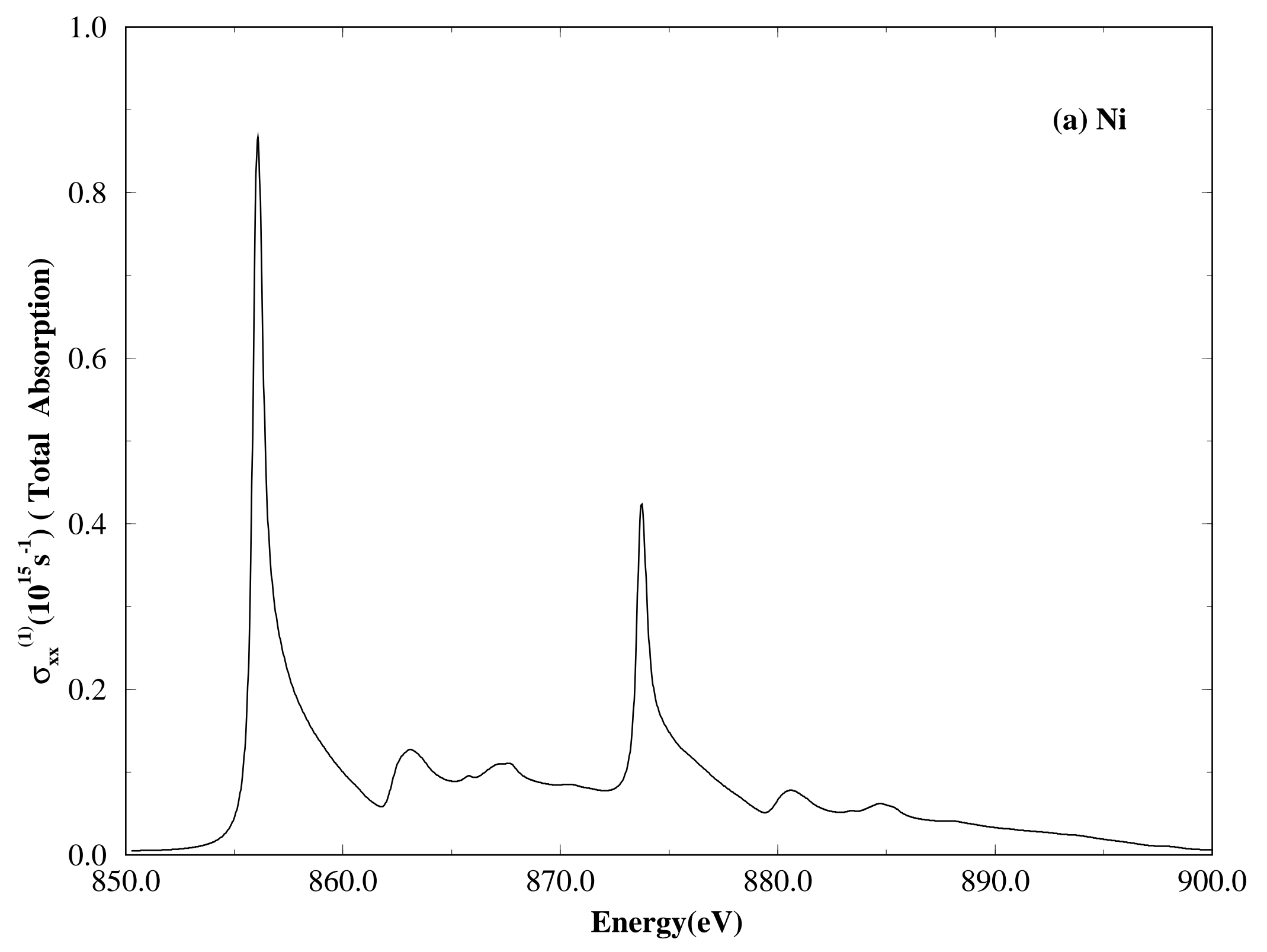




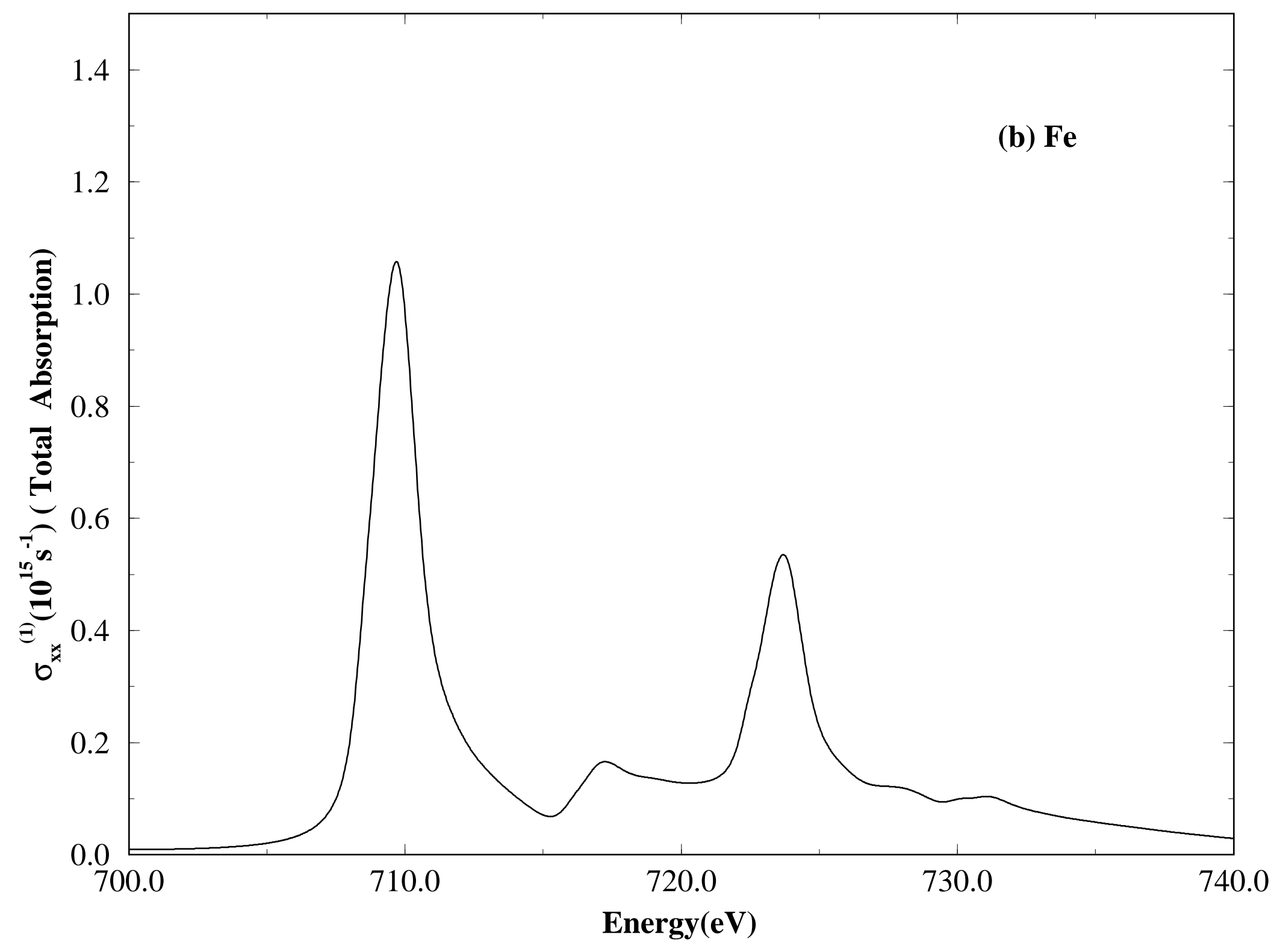




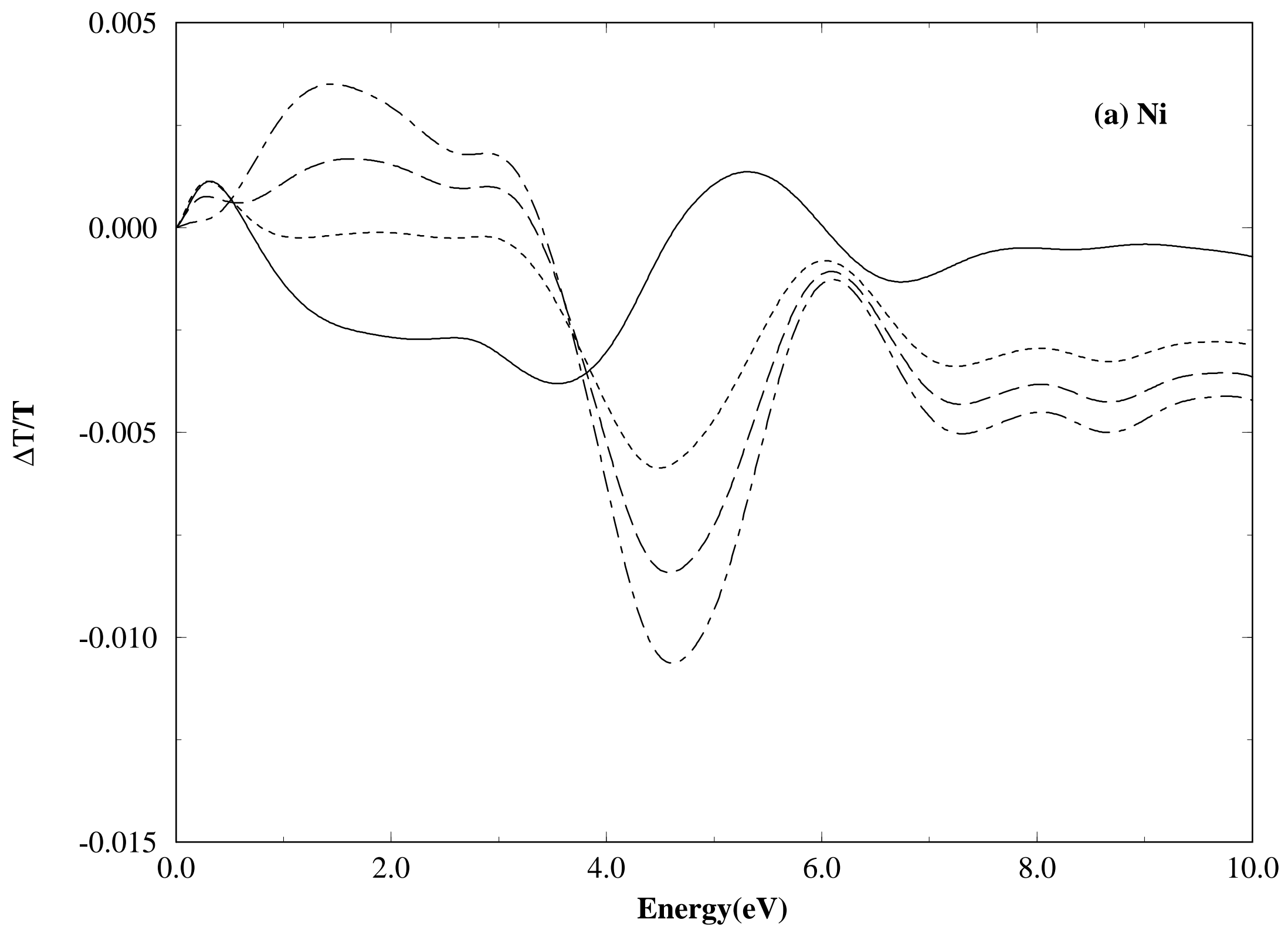




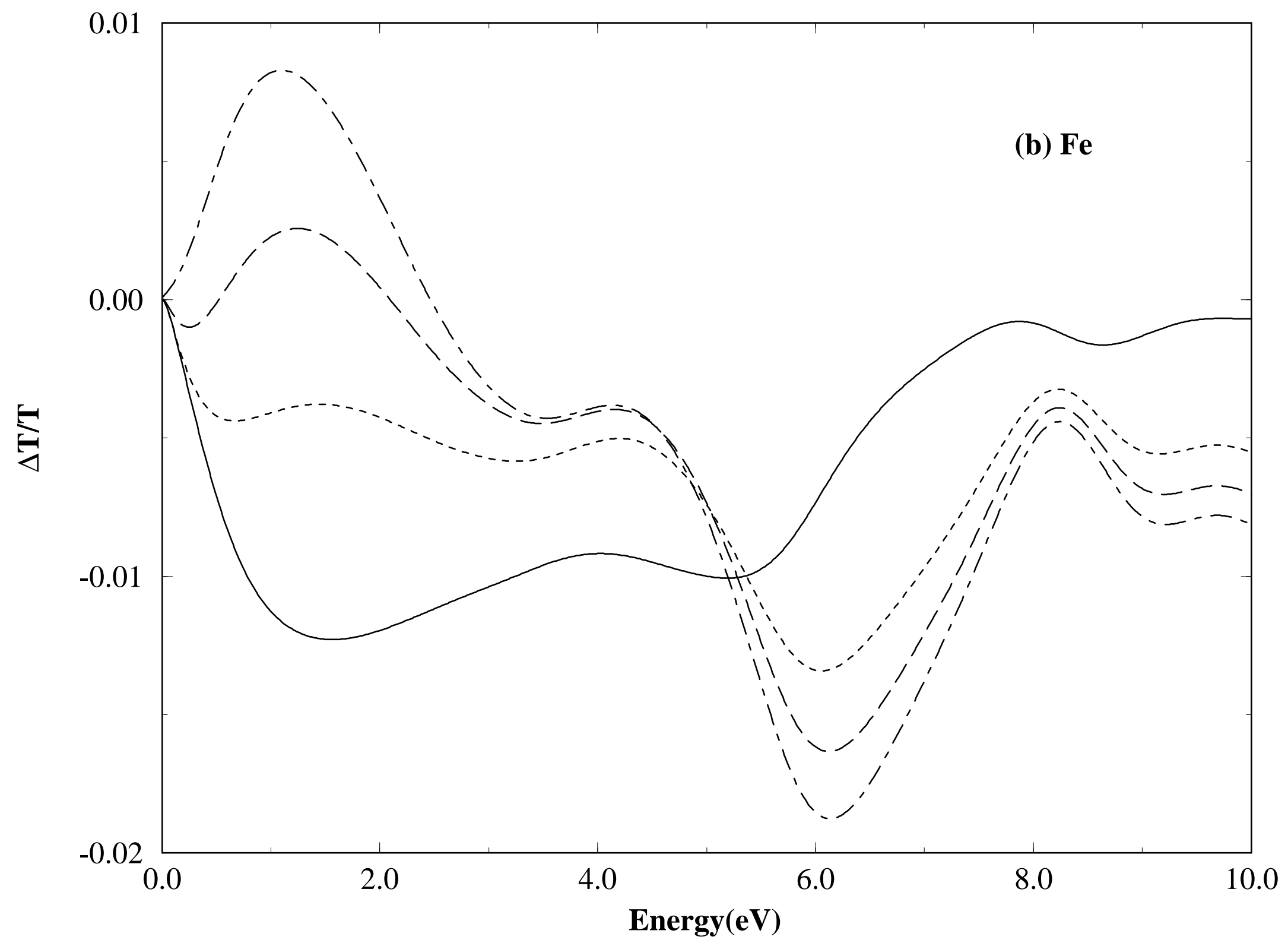




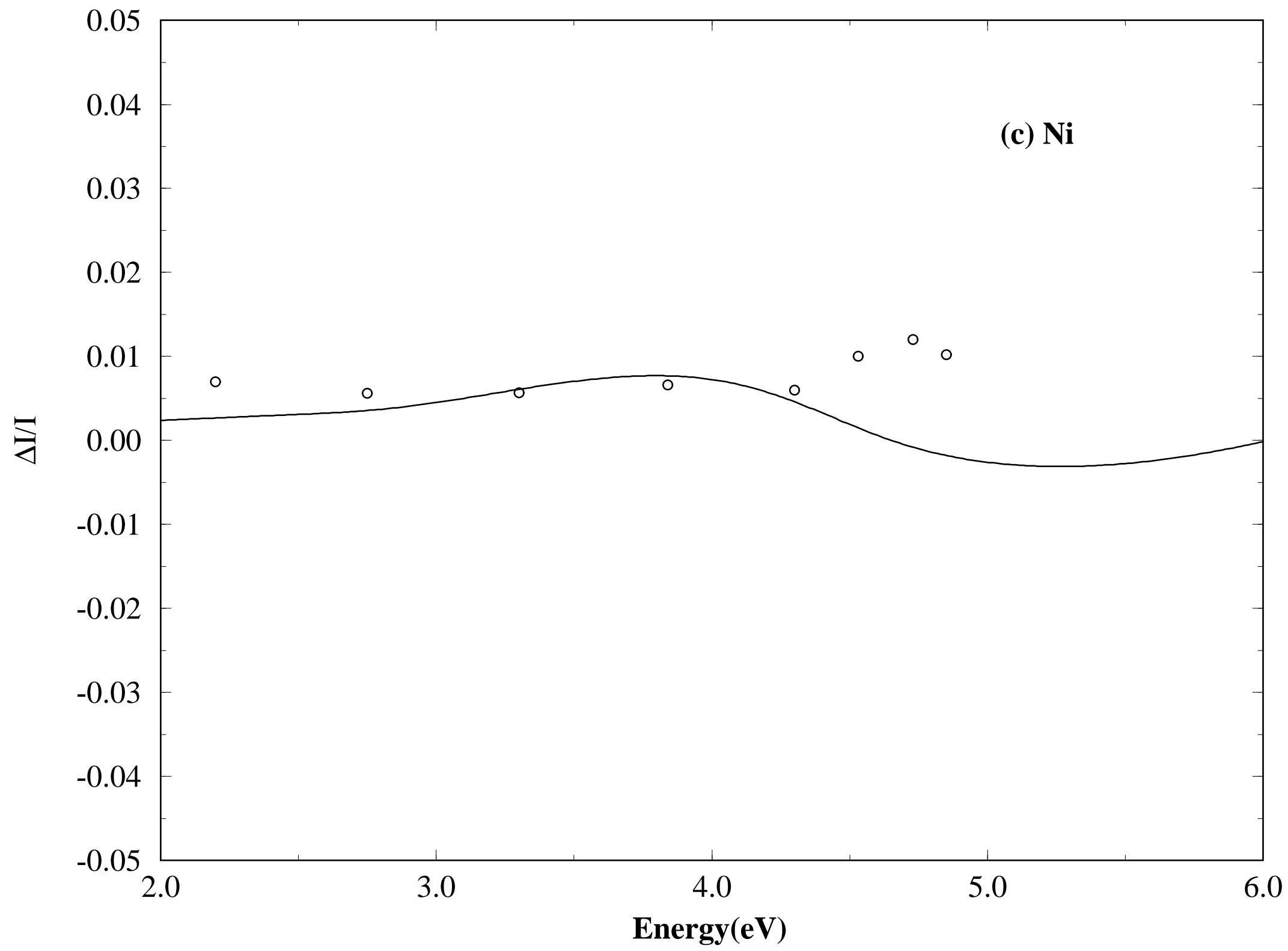




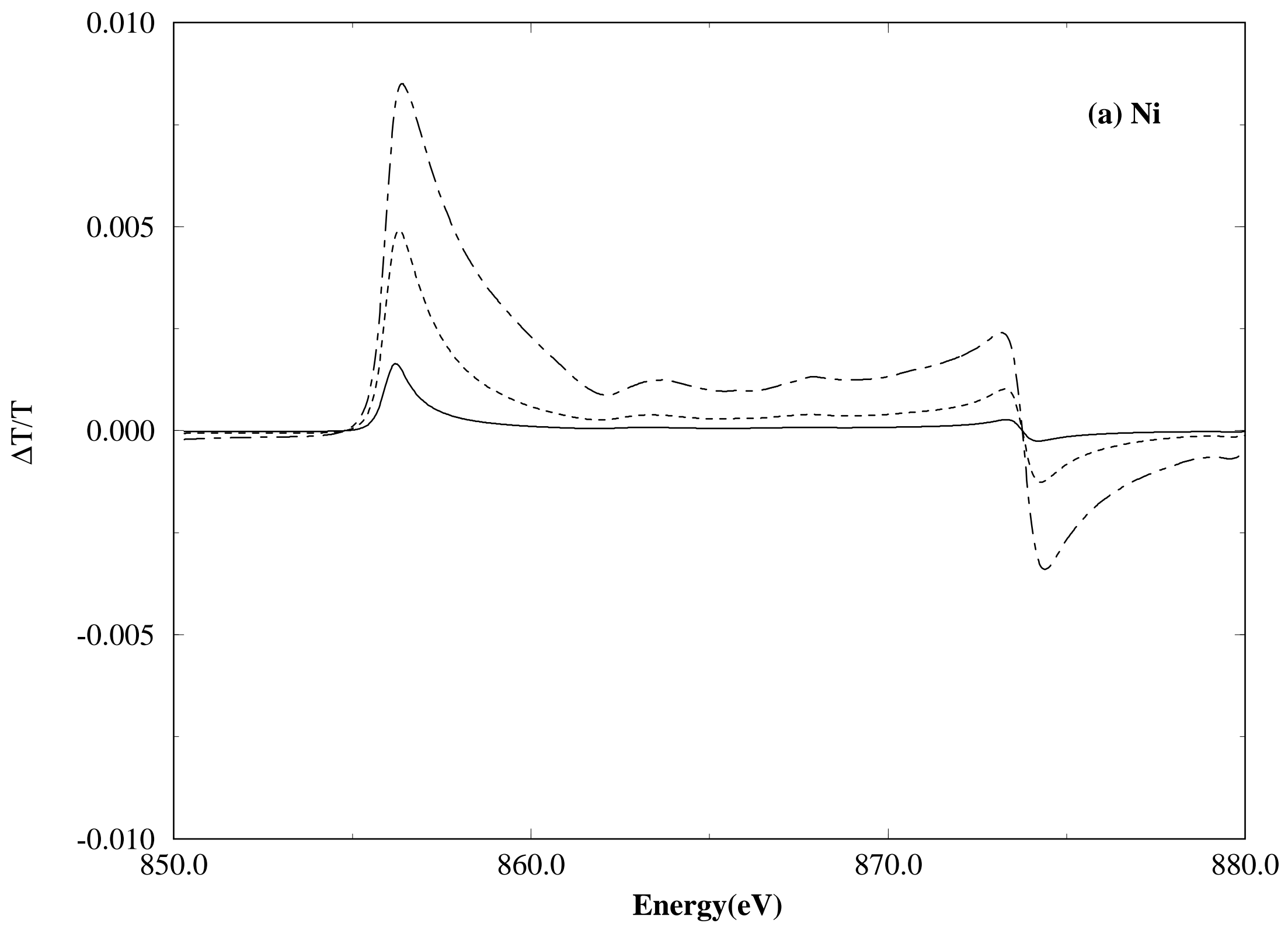




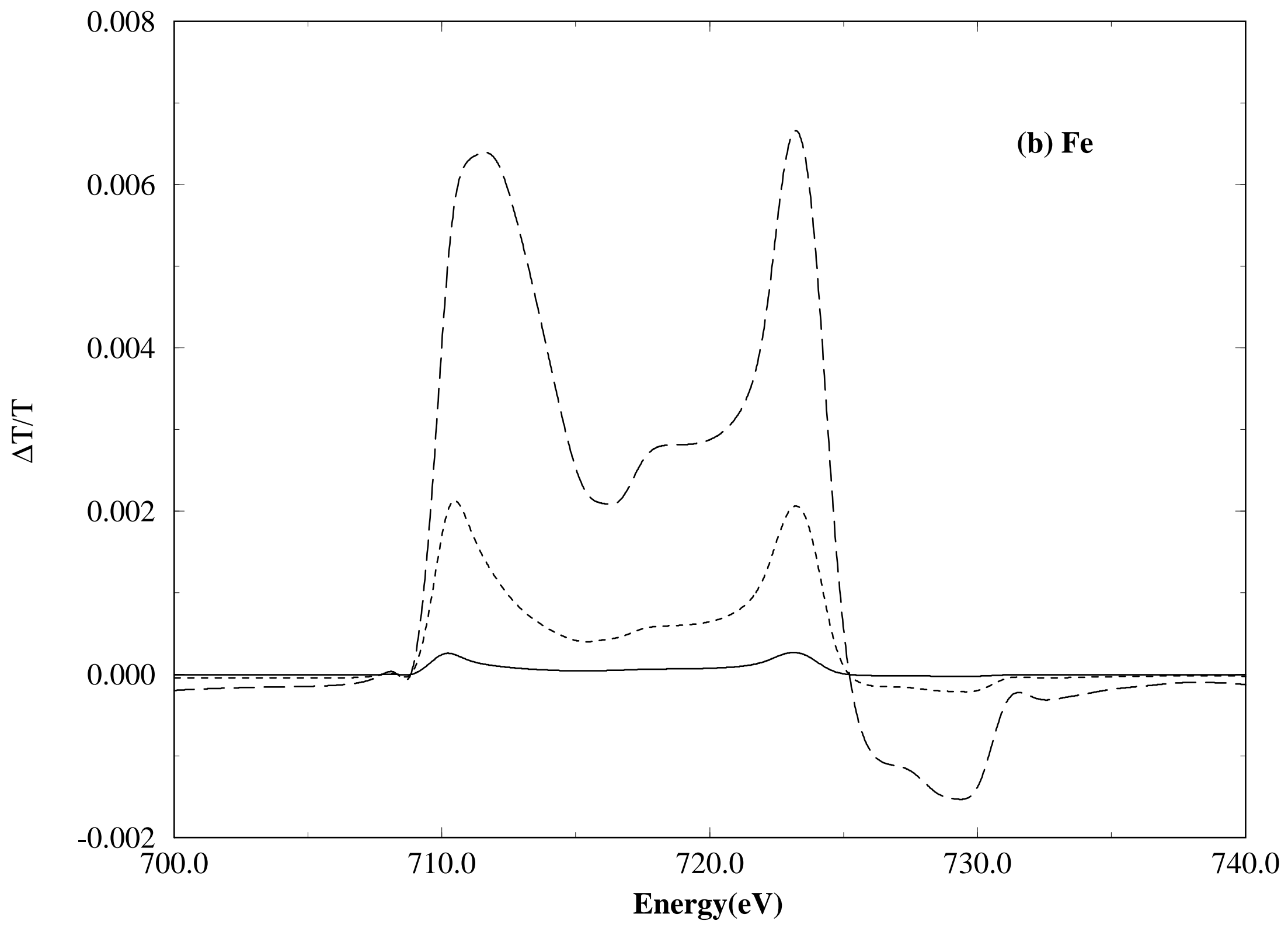




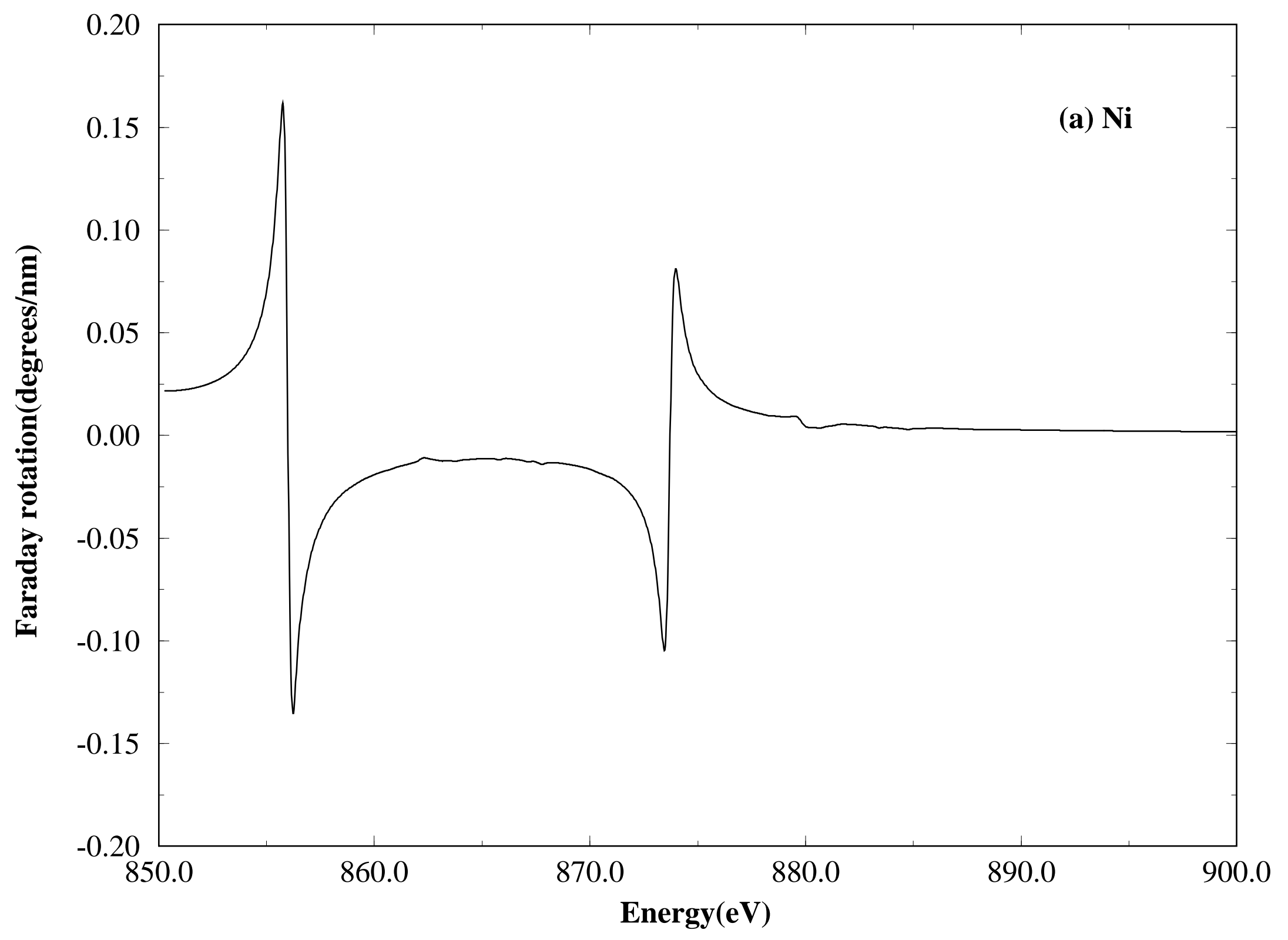




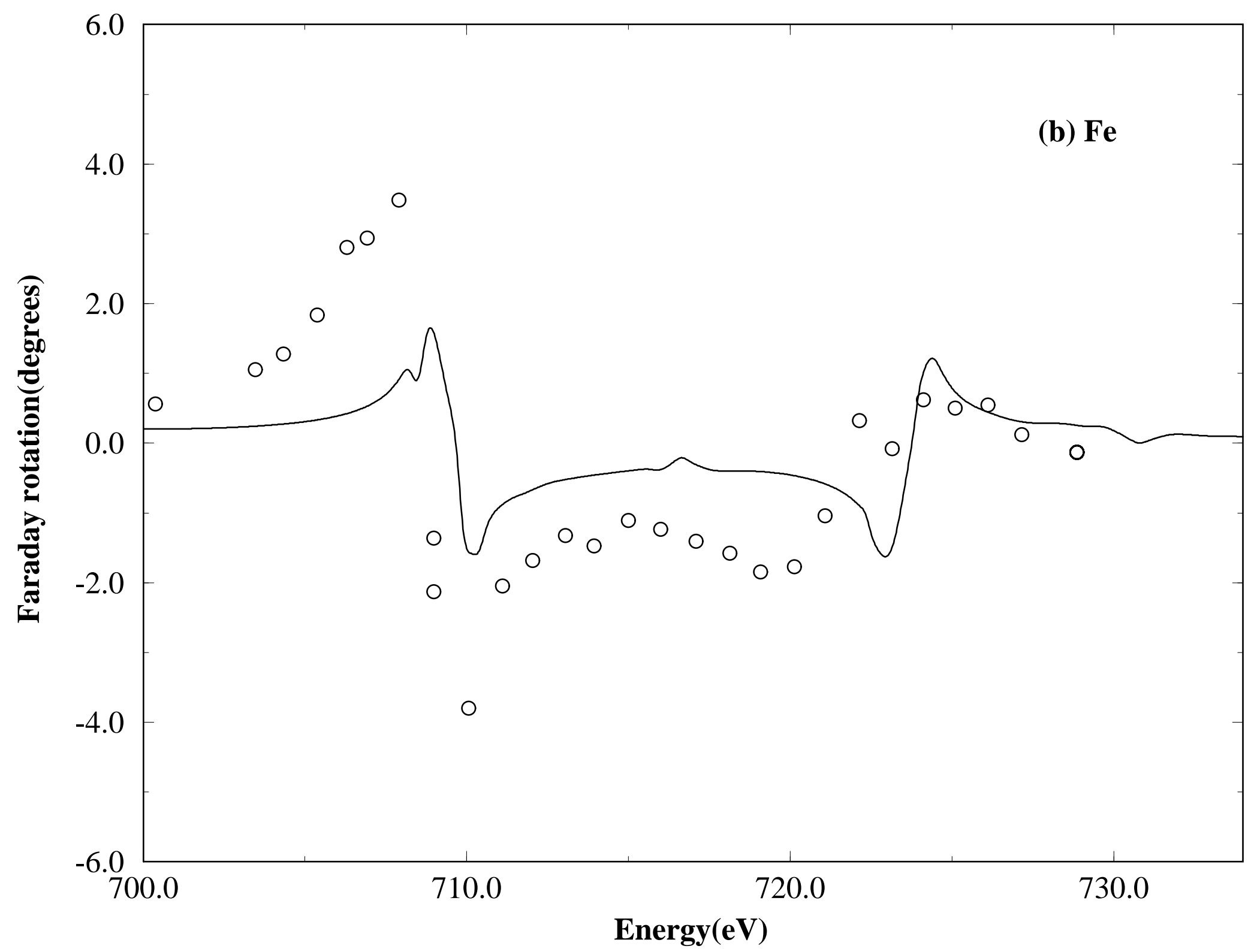

